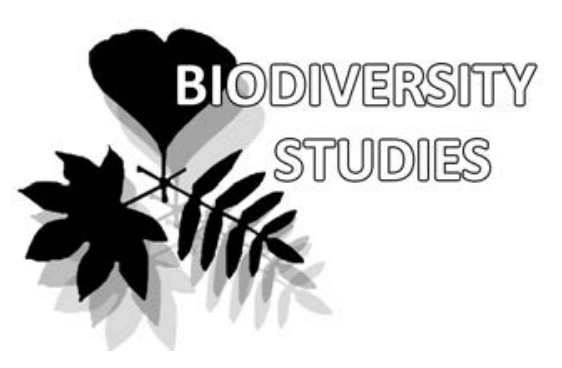

Vladimir I. Gmoshinskiy*

e-mail: rubisco@list.ru

Andrey V. Matveev

e-mail: andrmatveev@gmail.com

Evgeny S. Gubanov

e-mail: gubanov_5b_1315@mail.ru

Fedor M. Bortnikov

e-mail: f.m.bortnikov@gmail.com

Evgeny A. Dunayev

e-mail: dunayeve@mail.ru

Lomonosov Moscow State University, Moscow, Russia

* corresponding author

Manuscript received: 17.05 .2020

Review completed: 24.08 .2020

Accepted for publication: 28.08 .2020

Published online: 01.09.2020

\section{Critical revision of the Myxomycetes collection of Young Naturalists Club of Zoological Museum of Moscow State University}

Vladimir I. Gmoshinskiy*, Andrey V. Matveev, Evgeny S. Gubanov, Fedor M. Bortnikov \& Evgeny A. Dunayev

\begin{abstract}
A B S T R A C T
In the present paper, we report the results of a critical revision of the Myxomycetes collection of Young Naturalists Club of Zoological Museum of Moscow State University. The collection consists of 1715 specimens from 142 species in 35 genera, 11 families, and 6 orders. Comprehensive material from Moscow and Moscow Region (1112 specimens), Tver Region (191), and Tyumen Region (112) is presented in the collection. There are also numerous specimens from Irkutsk, Murmansk, Ryazan Regions, and Primorye Territory, and fragmentary collections from Astrakhan, Kaluga, Pskov, Vladimir, Yaroslavl Regions, Altai, Krasnodar, Khabarovsk Territories, Altai and Karelia Republics, as well as Crimean Peninsula and Kingdom of Cambodia (Southeast Asia). During revision we discovered two new species for Russia: Stemonitis wifera T. Macbr. and Fuligo intermedia T. Macbr. Descriptions and illustrations of these species are presented.
\end{abstract}

Ke y w o r d s : Amoebozoa, biodiversity, slime moulds, herbarium, Russia

\section{P Е 3 Ю М E}

Гмошинский В.И., Матвеев А.В., Губанов Е.С., Бортников Ф.М., Аунаев Е.А. Критическая ревизия коммекции миксомицетов Кружка юных натуралистов Зоологического музея МГУ. В работе приведены результаты ревизии колмекции миксомицетов (Amoebozoa, Myxomycota) Кружка юных натуралистов Зоологического музея МГУ. Она вкАючает 1715 образцов спороношений, относящихся к 142 видам миксомицетов из 35 родов, 11 семейств и 6 порядков. В колмекции обширно представлены материалы, собранные на территории Московской (1112 образцов), Тверской (191) и Тюменской (112) областей. Аепонировано значительное число образцов из Иркутской, Мурманской, Рязанской областей и Приморского края, присутствуют фрагментарные сборы из Астраханской, ВАацимирской, Кацужской, Псковской и Ярославской областей, А^тайского, Краснодарского и Хабаровского краев, республик Алтай, Карелия и Крым, а также из Королевства Камбоожа (Юго-Восточная Азия). При ревизии комлекции были обнаружены два новых вила Аля России - Stemonitis wvifera T. Macbr. и Fuligo intermedia T. Macbr., их ил^юстрации и описания особенностей морфологии приведены в статье.

КАючевые слова: Amoebozoа, биоразнообразие, гербарий, Россия, слизевики
The myxomycetes (Amoebozoa, Myxogastrea) are a relatively small group of amoeboid fungus-like protists which are widely spread across all continents (Schnittler et al. 2017). According to the online nomenclatural information system of Eumycetozoa (Lado 2005-2020), this group consists of approximately 1050 morphospecies. Some of them apparently occur more often in certain terrestrial ecoregions (Novozhilov et al. 2009). It is important to note that myxomycetes are a very convenient object for herbarium storage. The specimens of fruit bodies (sporophores) on substrate pieces are relatively small (mostly in the range of 10-100 mm) and take up little space in herbarium. Unlike macromycete fungi, it is not significant on which substrate myxomycete specimens were found. Almost all essential information to identify myxomycete species can be obtained from morphological characters of sporophores.

Despite the research intensification over the last 40 years, the territory of Russia remains extremely unevenly studied. The most well-studied areas are Astrakhan, Volgograd, Leningrad, Moscow, Novosibirsk, Sverdlovsk, and Tver Regions, Altai and Krasnoyarsk Territories, and Republic of Karelia. A total of 454 myxomycete species from 56 genera and 6 orders are found in Russia, which is approximately $42 \%$ of the total number of known species (Matveev et al. 2016-2020). However, studies of species diversity have never been conducted in most areas of Russia. Therefore, critical revisions of the available collections may provide additional information on the distribution of certain species throughout the country.

The Club of Young Naturalists of Zoological Museum of Moscow State University (CYN) was founded by Evgeny A. Dunayev in 1991. Since then, more than 512 students have been trained in this club and many of them have subsequently chosen biology as their main area of professional activity. During the years of the CYN existence, more than 135 field courses have been conducted in Russia and other countries 
(Dunayev 2017). The Myxomycetes collection of CYN was created in 1992 by E.A. Dunayev, who is still maintaining it. Specimens collected during field courses represent the basis of the deposited material (Dunayev \& Barsukova 2002). In addition, part of the specimens was provided by the staff of the Department of Mycology and Algology (Biological Faculty, Lomonosov Moscow State University).

\section{MATERIAL AND METHODS}

Before the revision, all specimens in the collection were kept in matchboxes provided with finishing labels. A cata$\log$ containing the following information about the specimens was attached to the collection: taxon name and its authors; location description; date of collection; names of the collector and the person who identified the specimen. Sporophores of the same species collected on the same day and in the same habitat were often consolidated into one herbarium specimen, which was noted in the catalog.

During revision, all specimens were re-identified using widely accepted monographs (Martin \& Alexopoulos 1969, Novozhilov 1993, Poulain et al. 2011a). Taxa names and authors correspond to the online nomenclatural information system of Eumycetozoa (Lado 2005-2020). We used the classification of Lado \& Eliasson (2017). The level of preservation on a five-point scale was indicated for each specimen (according to dela Cruz et al. 2009, with modifications) (Table 1, see also Fig. 1). During identification, we attached them to the bottom of a U-shaped paper tray (according to Stephenson \& Stempen 2000). Labels with specimen unique number, species name, collection location and date, collector's and determination person's names were affixed to box tops.

Based on the analysis of the verbal description of collection sites, we assigned georeferences and location uncertainties in meters to all specimens.

All information about the specimens in the collection was modified according to the Darwin Core standard (Wieczorek et al. 2012) and presented as a dataset on gbif.org (Gmoshinskiy et al. 2018).

\section{List of collection sites}

\section{Altai Republic}

1. $51.762990^{\circ} \mathrm{N} 85.732476^{\circ} \mathrm{E}( \pm 1 \mathrm{~km}), 06.08 .2007$. Altai Territory

2. $52.569319^{\circ} \mathrm{N} 78.928442^{\circ} \mathrm{E}( \pm 1 \mathrm{~km}), 05.08 .2007$.

Table 1. Criteria adopted to establish the level of preservation of Myxomycetes collection of CYN

\begin{tabular}{ll}
\hline Level & Description \\
\hline I & $\begin{array}{l}\text { No traces of sporophores } \\
\text { II }\end{array}$ \\
$\begin{array}{l}\text { Sporophores are heavily damaged. Only stalks and } \\
\text { hypothallus of the sporophores are preserved. Precise } \\
\text { identification is impossible }\end{array}$ \\
III & $\begin{array}{l}\text { Sporophores are heavily damaged, but it is possible to } \\
\text { identify species by using a set of diagnostic features. } \\
\text { There are } \geq 10 \text { complete sporophores in collection }\end{array}$ \\
IV & $\begin{array}{l}\text { Sporophores in good condition, but many of them are } \\
\text { slightly damaged (in particular, broken peridium, whole } \\
\text { colonies are pressed as a result of incorrect storage, etc.) } \\
\text { Sporophores in excellent condition }\end{array}$ \\
V &
\end{tabular}

\section{Astrakhan Region}

3. $46.904181^{\circ} \mathrm{N} 47.913736^{\circ} \mathrm{E}( \pm 5 \mathrm{~km})$, a. 06.08.1999, b. 08.2005 .

\section{Crimean Peninsula}

4. $44.508184^{\circ} \mathrm{N} 34.236980^{\circ} \mathrm{E}( \pm 10 \mathrm{~m}), 03.02 .1999$.

5. $44.508199^{\circ} \mathrm{N} 34.241218^{\circ} \mathrm{E}( \pm 30 \mathrm{~m}), 30.04 .2013$.
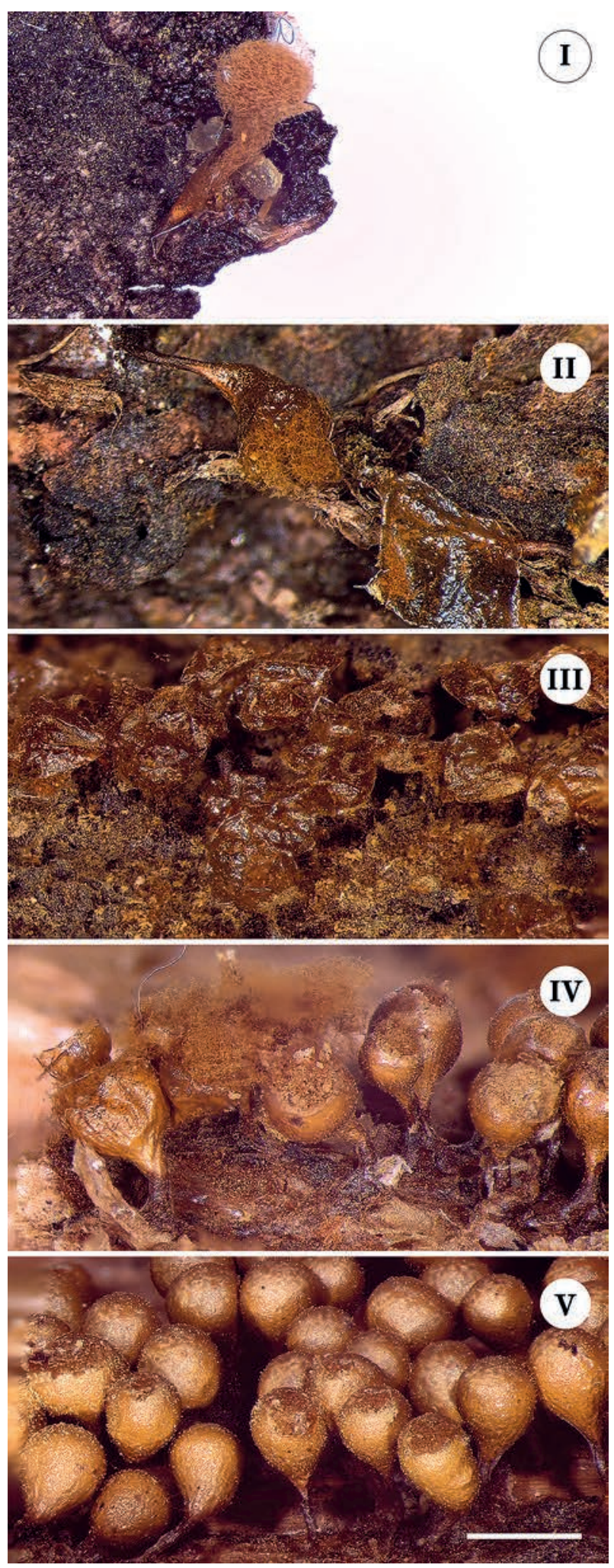

Figure 1 Specimens of Tricbia decipiens (Pers.) T. Macbr. with levels of preservation from I to V. Scale bar: $1 \mathrm{~mm}$ 
6. $44.508390^{\circ} \mathrm{N} 34.240043^{\circ} \mathrm{E}( \pm 30 \mathrm{~m})$, collection date is unknown.

7. $44.531043^{\circ} \mathrm{N} 34.255920^{\circ} \mathrm{E}( \pm 50 \mathrm{~m}), 17.06 .1999$.

8. $44.561064^{\circ} \mathrm{N} 34.327681^{\circ} \mathrm{E}( \pm 20 \mathrm{~m}), 03.05 .2002$.

9. $44.644440^{\circ} \mathrm{N} 34.370180^{\circ} \mathrm{E}( \pm 50 \mathrm{~m}), 02.04 .1997$.

10. $44.661576^{\circ} \mathrm{N} 34.346115^{\circ} \mathrm{E}( \pm 20 \mathrm{~m}), 02.04 .1997$.

11. $44.666132^{\circ} \mathrm{N} 34.336719^{\circ} \mathrm{E}( \pm 500 \mathrm{~m}), 04.05 .2002$.

12. $44.757732^{\circ} \mathrm{N} 34.345456^{\circ} \mathrm{E}( \pm 30 \mathrm{~m}), 06.05 .2002$.

13. $44.766951^{\circ} \mathrm{N} 34.392726^{\circ} \mathrm{E}( \pm 70 \mathrm{~m}), 08.05 .2003$.

14. Exact collection locality and collection date are unknown.

\section{Irkutsk Region}

15. $51.8970987^{\circ} \mathrm{N} 105.0548744^{\circ} \mathrm{E}( \pm 1 \mathrm{~km}), 02.06 .2009$.

16. $52.030049^{\circ} \mathrm{N} 104.638495^{\circ} \mathrm{E}( \pm 1 \mathrm{~km})$, a. 20.05 .2005 - 01.06.2005; b. 28.05.2006 - 15.06.2006; c. $24.05 .2007-30.06 .2007$; d. $16.05 .2008-$ 01.06 .2008 .

17. Exact collection locality is unknown; 2006.

\section{Kaluga Region}

18. $54.553557^{\circ} \mathrm{N} 36.328644^{\circ} \mathrm{E}(+5 \mathrm{~km}), 10.10 .1998$.

19. $54.732455^{\circ} \mathrm{N} 35.233281^{\circ} \mathrm{E}( \pm 5 \mathrm{~m}), 12.09 .2011$.

\section{Khabarovsk Territory}

20. $48.528895^{\circ} \mathrm{N} 135.025458^{\circ} \mathrm{E}( \pm 1 \mathrm{~km}), 27.09 .2005$.

\section{Krasnodar Territory}

21. $44.700234^{\circ} \mathrm{N} 37.593121^{\circ} \mathrm{E}( \pm 1 \mathrm{~km}), 12.07 .2010$. 22. $44.730479^{\circ} \mathrm{N} 37.434199^{\circ} \mathrm{E}( \pm 100 \mathrm{~m}), 28.03 .2003$ 23. $44.750953^{\circ} \mathrm{N} 37.411057^{\circ} \mathrm{E}( \pm 100 \mathrm{~m}), 27.03 .2003$. 24. $44.771465^{\circ} \mathrm{N} 37.405274^{\circ} \mathrm{E}( \pm 50 \mathrm{~m}), 25.03 .2001$. 25. $44.771541^{\circ} \mathrm{N} 37.392413^{\circ} \mathrm{E}( \pm 50 \mathrm{~m}), 26.03 .2003$.

\section{Moscow and Moscow Region}

26. $54.418306^{\circ} \mathrm{N} 38.516885^{\circ} \mathrm{E}( \pm 5 \mathrm{~km}), 07.08 .1994$. 27. $54.473926^{\circ} \mathrm{N} 38.724988^{\circ} \mathrm{E}( \pm 5 \mathrm{~km}), 07.08 .1994$.

28. $54.771368^{\circ} \mathrm{N} 37.599469^{\circ} \mathrm{E}( \pm 1 \mathrm{~km}), 08.06 .2002$.

29. $54.884892^{\circ} \mathrm{N} 37.617438^{\circ} \mathrm{E}( \pm 5 \mathrm{~km})$, a. 03.05.1993; b. 17.06.1993-12.08.1993; c. 06.10.1993; d. 27.05.1994; e. 08.01.2018; f. 1993.

30. $54.984049^{\circ} \mathrm{N} 38.194669^{\circ} \mathrm{E}( \pm 1 \mathrm{~km}), 03.05 .1997$. 31. $55.317027^{\circ} \mathrm{N} 37.137380^{\circ} \mathrm{E}( \pm 1 \mathrm{~km}), 23.10 .2003$.

32. $55.318798^{\circ} \mathrm{N} 36.913751^{\circ} \mathrm{E}( \pm 5 \mathrm{~km}), 08.09 .1991$.

33. $55.414201^{\circ} \mathrm{N} 38.574336^{\circ} \mathrm{E}( \pm 50 \mathrm{~m}), 22.04 .1995$.

34. $55.445185^{\circ} \mathrm{N} 35.987815^{\circ} \mathrm{E}( \pm 100 \mathrm{~m}), 1991$.

35. $55.448703^{\circ} \mathrm{N} 36.002789^{\circ} \mathrm{E}( \pm 1 \mathrm{~km})$, a. 23.04 .1989 ; b. 02.05.1990; c. 06.08.1990; d. 06.10.1991.

36. $55.453369^{\circ} \mathrm{N} 35.968434^{\circ} \mathrm{E}( \pm 30 \mathrm{~m}), 11.04 .1993$.

37. $55.462264^{\circ} \mathrm{N} 40.031258^{\circ} \mathrm{E}( \pm 1 \mathrm{~km}), 26.07 .1994-$ 29.07 .1994

38. $55.452248^{\circ} \mathrm{N} 40.030098^{\circ} \mathrm{E}( \pm 1 \mathrm{~km}), 29.07 .1994$

39. $55.471299^{\circ} \mathrm{N} 40.030457^{\circ} \mathrm{E}( \pm 1 \mathrm{~km}), 29.07 .1994$.

40. $55.481450^{\circ} \mathrm{N} 40.014083^{\circ} \mathrm{E}( \pm 1 \mathrm{~km}), 27.07 .1994$.

41. $55.481607^{\circ} \mathrm{N} 40.030295^{\circ} \mathrm{E}( \pm 1 \mathrm{~km}), 27.07 .1994$.

42. $55.483014^{\circ} \mathrm{N} 38.444712^{\circ} \mathrm{E}( \pm 1 \mathrm{~km}), 31.05 .1995$.

43. $55.490276^{\circ} \mathrm{N} 38.444283^{\circ} \mathrm{E}( \pm 1 \mathrm{~km}), 02.08 .1994$

44. $55.491720^{\circ} \mathrm{N} 38.466306^{\circ} \mathrm{E}( \pm 1 \mathrm{~km}), 02.08 .1994$.

45. $55.492067^{\circ} \mathrm{N} 38.435565^{\circ} \mathrm{E}( \pm 1 \mathrm{~km})$, a. 13.09 .1990 ; b. 07.01 .1991 ; c. 23.08.1992; d. 1989; e. 1990; f. 1991.

46. $55.511765^{\circ} \mathrm{N} 36.221113^{\circ} \mathrm{E}( \pm 1 \mathrm{~km})$, a. 16.09 .1990 ; b. 05.09 .1991 .

47. $55.535992^{\circ} \mathrm{N} 36.589461^{\circ} \mathrm{E}( \pm 1 \mathrm{~km}), 19.04 .2014$.

48. $55.565815^{\circ} \mathrm{N} 38.856443^{\circ} \mathrm{E}( \pm 50 \mathrm{~m}), 09.2002$

49. $55.570593^{\circ} \mathrm{N} 38.832239^{\circ} \mathrm{E}( \pm 10 \mathrm{~m}), 03.10 .1999$.

50. $55.571482^{\circ} \mathrm{N} 38.823449^{\circ} \mathrm{E}( \pm 20 \mathrm{~m}), 28.09 .2014$.

51. $55.572144^{\circ} \mathrm{N} 38.842642^{\circ} \mathrm{E}( \pm 50 \mathrm{~m}), 22.09 .1996$.

52. $55.572833^{\circ} \mathrm{N} 37.784500^{\circ} \mathrm{E}( \pm 5 \mathrm{~m}), 29.05 .2010$.

53. $55.579058^{\circ} \mathrm{N} 36.587530^{\circ} \mathrm{E}( \pm 1 \mathrm{~km})$, a. 24.03 .1991 ; b. $22.03 .1992-26.03 .1992$

54. $55.581653^{\circ} \mathrm{N} 38.755459^{\circ} \mathrm{E}( \pm 1 \mathrm{~km}), 19.06 .1993$.

55. $55.569260^{\circ} \mathrm{N} 38.836419^{\circ} \mathrm{E}( \pm 20 \mathrm{~m})$, a. 11.10 .1992 ; b. 25.09 .2014 .

56. $55.590827^{\circ} \mathrm{N} 38.858224^{\circ} \mathrm{E}( \pm 1 \mathrm{~km}), 10.10 .1993$.

57. $55.596667^{\circ} \mathrm{N} 37.751667^{\circ} \mathrm{E}( \pm 5 \mathrm{~m}), 26.09 .2010$

$$
-29.09 .2010 \text {. }
$$

58. $55.599971^{\circ} \mathrm{N} 37.740464^{\circ} \mathrm{E}( \pm 5 \mathrm{~m}), 14.07 .2007$.

59. $55.608674^{\circ} \mathrm{N} 37.689313^{\circ} \mathrm{E}( \pm 100 \mathrm{~m}), 20.12 .2009$.

60. $55.612851^{\circ} \mathrm{N} 37.684122^{\circ} \mathrm{E}( \pm 500 \mathrm{~m}), 23.03 .1991$.

61. $55.601422^{\circ} \mathrm{N} 37.925407^{\circ} \mathrm{E}( \pm 5 \mathrm{~km}), 14.06 .2010$.

62. $55.622483^{\circ} \mathrm{N} 37.727228^{\circ} \mathrm{E}( \pm 5 \mathrm{~m}), 15.10 .2011$.

63. $55.624000^{\circ} \mathrm{N} 37.727283^{\circ} \mathrm{E}( \pm 5 \mathrm{~m}), 30.10 .2010$.

64. $55.617624^{\circ} \mathrm{N} 37.564978^{\circ} \mathrm{E}( \pm 5 \mathrm{~km})$, a. 10.09 .2000 - 24.09.2000; b. 10.09.2001; c. 18.10.2001. d. 06.04.2010; e. 28.06.2010; f. 09.06.2014.

65. $55.630133^{\circ} \mathrm{N} 38.195701^{\circ} \mathrm{E}( \pm 10 \mathrm{~m})$, a. 23.09.2001; b. 2005 .

66. $55.631283^{\circ} \mathrm{N} 37.581283^{\circ} \mathrm{E}( \pm 5 \mathrm{~m}), 09.06 .2010$.

67. $55.632174^{\circ} \mathrm{N} 38.203141^{\circ} \mathrm{E}( \pm 50 \mathrm{~m})$, a. 08.12 .1990 - 09.12.1990; b. 24.11.1991; c. 20.09.1992; d. 19.10.2008; e. 1990 .

68. $55.619200^{\circ} \mathrm{N} 38.612091^{\circ} \mathrm{E}( \pm 30 \mathrm{~m})$, collection date is unknown.

69. $55.646343^{\circ} \mathrm{N} 37.315080^{\circ} \mathrm{E}( \pm 1 \mathrm{~km})$, a. 04.04 .2004 ; b. 12.07 .2010 .

70. $55.647200^{\circ} \mathrm{N} 37.679500^{\circ} \mathrm{E}( \pm 5 \mathrm{~m}), 29.06 .2010$.

71. $55.658815^{\circ} \mathrm{N} 37.549875^{\circ} \mathrm{E}( \pm 1 \mathrm{~km}), 24.10 .2006$.

72. $55.659927^{\circ} \mathrm{N} 37.815521^{\circ} \mathrm{E}( \pm 1 \mathrm{~km}), 04.06 .2010$.

73. $55.651460^{\circ} \mathrm{N} 37.542904^{\circ} \mathrm{E}( \pm 5 \mathrm{~m})$, a. 10.05.2006; b. 05.10 .2006

74. $55.674941^{\circ} \mathrm{N} 37.693630^{\circ} \mathrm{E}( \pm 5 \mathrm{~m}), 29.06 .2010$.

75. $55.676526^{\circ} \mathrm{N} 36.925951^{\circ} \mathrm{E}( \pm 1 \mathrm{~km})$, a. 03.10.1999; b. 24.09 .2000

76. $55.678365^{\circ} \mathrm{N} 36.912055^{\circ} \mathrm{E}( \pm 50 \mathrm{~m}), 28.09 .1997$.

77. $55.678744^{\circ} \mathrm{N} 36.964902^{\circ} \mathrm{E}( \pm 100 \mathrm{~m})$, a. 23.09 .1990 - 25.09.1990; b. 03.09.1991; c. 07.10.2001; d. 09.1991 .

78. $55.681233^{\circ} \mathrm{N} 36.727995^{\circ} \mathrm{E}( \pm 5 \mathrm{~km})$, a. 19.07 .1985 ; b. 02.07.1990 - 07.07.1990; c. 12.04.1991; d. 12.04.1992; e. 02.07.1992; f. 18.08.1992 10.09.1992; g. 02.07.1993 - 29.09.1993; h. $01.07 .1995-01.08 .1995$; i. 17.08.1996 06.11.1996; j. 06.06.1997 - 26.07.1997; k. 28.08.1997 - 13.10.1997; 1. 20.08.1998 - 24.08.1998; m. 25.09.1998 - 27.09.1998; n. 20.08.1999 22.08.1999; o. 01.07.2003 - 24.07.2003; p. 11.07.2007; q. 21.06.2010-25.06.2010; r. 01.10.2011; s. 1975 ; t. 1982; u. 1985; v. 09.1992; w. $1993 ; \mathbf{x} .08 .1993-09.1993 ;$ y. 06.1995.

79. $55.697778^{\circ} \mathrm{N} 38.412778^{\circ} \mathrm{E}( \pm 1 \mathrm{~km}), 11.12 .1995$

80. $55.685148^{\circ} \mathrm{N} 37.070238^{\circ} \mathrm{E}( \pm 1 \mathrm{~km}), 1993$.

81. $55.707283^{\circ} \mathrm{N} 37.527008^{\circ} \mathrm{E}( \pm 1 \mathrm{~km})$, a. 25.09 .2000 -05.10 .2000 ; b. 20.06.2001.

82. $55.710372^{\circ} \mathrm{N} 37.534850^{\circ} \mathrm{E}( \pm 1 \mathrm{~km}), 22.09 .2008$

83. $55.716992^{\circ} \mathrm{N} 37.587411^{\circ} \mathrm{E}( \pm 1 \mathrm{~km})$, a. 30.09 .2003 -12.10 .2003 ; b. 22.09 .2008

84. $55.719966^{\circ} \mathrm{N} 38.378226^{\circ} \mathrm{E}( \pm 5 \mathrm{~km}), 05.01 .1996$.

85. $55.753240^{\circ} \mathrm{N} 38.120674^{\circ} \mathrm{E}( \pm 1 \mathrm{~km})$, a. 15.08 .1991 - 25.08.1991; b. 13.04.2002; c. 14.10.2004; d. 15.09.2005; e. 28.10.2006 - 10.11.2006; f. 27.10.2013; g. collection date is unknown.

86. $55.759299^{\circ} \mathrm{N} 37.424102^{\circ} \mathrm{E}( \pm 1 \mathrm{~km}), 15.09 .2001$

87. $55.772867^{\circ} \mathrm{N} 37.677957^{\circ} \mathrm{E}( \pm 20 \mathrm{~m}), 03.07 .2013$.

88. $55.776631^{\circ} \mathrm{N} 37.782409^{\circ} \mathrm{E}( \pm 1 \mathrm{~km}), 10.05 .2010$ -10.06 .2010 .

89. $55.782375^{\circ} \mathrm{N} 38.115931^{\circ} \mathrm{E}( \pm 5 \mathrm{~km})$, a. 14.09 .1998 ; b. 15.09.2000; c. 24.07 .2002 ; d. 20.08.200306.09.2003; e. 16.09.2004-23.09.2004; f. 02.09 .2005 ; g. 15.06.2006; h. 15.09.2006 22.09 .2006 ; i. 08.2000 ; j. collection date is unknown.

90. $55.782500^{\circ} \mathrm{N} 37.808250^{\circ} \mathrm{E}( \pm 5 \mathrm{~m}), 10.05 .2010$.

91. $55.811654^{\circ} \mathrm{N} 37.989802^{\circ} \mathrm{E}( \pm 1 \mathrm{~km}), 10.2006$.

92. $55.846539^{\circ} \mathrm{N} 37.740247^{\circ} \mathrm{E}( \pm 5 \mathrm{~km}), 25.08 .2003$.

93. $55.900875^{\circ} \mathrm{N} 37.742402^{\circ} \mathrm{E}( \pm 1 \mathrm{~km}), 10.1980$.

94. $55.910510^{\circ} \mathrm{N} 37.212507^{\circ} \mathrm{E}( \pm 1 \mathrm{~km})$, a. 29.10 .1989 ; b. 30.09 .1990 ; c. 29.01.1991; d. 22.09.1991 29.09.1991; e. 27.09.1992; f. 24.10.1993; g. 23.11 .1995 ; h. 14.09 .1997 ; i. 01.10 .2006 ; j. 23.09 .2012 .

95. $55.921475^{\circ} \mathrm{N} 36.731316^{\circ} \mathrm{E}( \pm 1 \mathrm{~km}), 16.10 .1994$

96. $55.926186^{\circ} \mathrm{N} 36.716532^{\circ} \mathrm{E}( \pm 1 \mathrm{~km})$, a. 24.09 .1995 ; b. 19.07 .1997 ; c. 19.10 .1997 .

97. $55.917861^{\circ} \mathrm{N} 37.218860^{\circ} \mathrm{E}( \pm 50 \mathrm{~m}), 08.03 .1992$

98. $55.935644^{\circ} \mathrm{N} 37.166286^{\circ} \mathrm{E}( \pm 100 \mathrm{~m}), 12.1990$. 
99. $55.956280^{\circ} \mathrm{N} 37.122331^{\circ} \mathrm{E}( \pm 1 \mathrm{~km}), 07.10 .1990$.

100. $56.005126^{\circ} \mathrm{N} 36.323589^{\circ} \mathrm{E}( \pm 1 \mathrm{~km})$, a. 20.03 .1993 ; b. 06.11.1996 - 09.11.1996; c. 01.01.1997; d. $01.11 .1997-09.11 .1997$.

101. $56.005126^{\circ} \mathrm{N} 36.323589^{\circ} \mathrm{E}( \pm 5 \mathrm{~km}), 07.10 .1991$

102. $56.025464^{\circ} \mathrm{N} 38.429571^{\circ} \mathrm{E}( \pm 1 \mathrm{~km}), 04.11 .1991$ $-06.11 .1991$

103. $56.208728^{\circ} \mathrm{N} 37.006008^{\circ} \mathrm{E}( \pm 5 \mathrm{~km})$, collection date is unknown.

104. $56.043928^{\circ} \mathrm{N} 36.254317^{\circ} \mathrm{E}( \pm 1 \mathrm{~km}), 28.08 .1994$

105. $56.048835^{\circ} \mathrm{N} 37.492784^{\circ} \mathrm{E}( \pm 1 \mathrm{~km})$, a. 16.10 .1996 ; b. $19.07 .1998-12.09 .1998$.

106. $56.512152^{\circ} \mathrm{N} 37.544756^{\circ} \mathrm{E}(+1 \mathrm{~km}), 13.10 .1991$

107. $56.526293^{\circ} \mathrm{N} 37.550477^{\circ} \mathrm{E}( \pm 100 \mathrm{~m})$, a. 29.09 .1991 - 23.10.1991; b. 05.09.1993 - 27.09.1993; c. $12.09 .1994 ;$ d. $14.08 .1996-17.08 .1996$.

108. $56.677767^{\circ} \mathrm{N} 37.236967^{\circ} \mathrm{E}( \pm 50 \mathrm{~m}), 19.04 .1992$

109. $56.696654^{\circ} \mathrm{N} 37.586167^{\circ} \mathrm{E}( \pm 1 \mathrm{~km}), 04.08 .1996$ $-19.08 .1996$

110. $56.699621^{\circ} \mathrm{N} 37.196171^{\circ} \mathrm{E}( \pm 2 \mathrm{~km})$, a. 31.12 .1990 - 10.01.1991; b. 07.02.1992; c. 07.11.2001; d. 25.03.2002; e. 02.11.2003-07.11.2003; f. 02.11.2004-07.11.2004; g. 29.12.200505.01.2006; h. 05.11.2006 - 10.11.2006; i. 27.12.2006-31.12.2006; j. 15.07.2008; k. $24.06 .2011-30.06 .2011 ; 1.27 .12 .2011-$ 05.01.2012; m. 10.10.2013; n. 29.06.2014; o. 27.06.2015; p. 29.10.2017; q. 10.1999; r. 11.2004; s. $2005 ; \mathbf{t}$ collection date is unknown.

111. $56.706602^{\circ} \mathrm{N} 37.152319^{\circ} \mathrm{E}( \pm 50 \mathrm{~m}), 10.10 .2013$ -09.11 .2013 .

112. $56.701443^{\circ} \mathrm{N} 38.182965^{\circ} \mathrm{E}( \pm 1 \mathrm{~km})$, a. 23.05 .1994 -27.05 .1994 ; b. 20.07.1994.

113. $56.721446^{\circ} \mathrm{N} 38.082329^{\circ} \mathrm{E}( \pm 1 \mathrm{~km})$, a. 03.05 .1994 ; b. $23.07 .1994-23.08 .1994$.

114. $56.727899^{\circ} \mathrm{N} 37.622286^{\circ} \mathrm{E}( \pm 5 \mathrm{~km}), 03.08 .1996$

115. $56.093617^{\circ} \mathrm{N} 37.298502^{\circ} \mathrm{E}( \pm 50 \mathrm{~m}), 23.05 .1992$ $-24.05 .1992$

116. $56.094901^{\circ} \mathrm{N} 37.300509^{\circ} \mathrm{E}( \pm 50 \mathrm{~m}), 19.05 .1996$

117. $56.145966^{\circ} \mathrm{N} 36.781394^{\circ} \mathrm{E}( \pm 1 \mathrm{~km}), 24.06 .2011$.

118. Exact collection locality and collection date are unknown

\section{Murmansk Region}

119. $66.301711^{\circ} \mathrm{N} 36.409739^{\circ} \mathrm{E}( \pm 1 \mathrm{~km}), 03.09 .2003$.

120. $66.523452^{\circ} \mathrm{N} 35.099388^{\circ} \mathrm{E}( \pm 1 \mathrm{~km}), 31.08 .2003$.

121. $66.8893302^{\circ} \mathrm{N} 33.0365753^{\circ} \mathrm{E}( \pm 1 \mathrm{~km}), 17.09 .2003$.

122. $66.9462324^{\circ} \mathrm{N} 32.9123783^{\circ} \mathrm{E}( \pm 1 \mathrm{~km}), 01.09 .1998$.

123. $67.167729^{\circ} \mathrm{N} 31.992337^{\circ} \mathrm{E}( \pm 1 \mathrm{~km}), 23.07 .1996$ $-23.08 .1996$

124. $67.1854688^{\circ} \mathrm{N} 32.9265404^{\circ} \mathrm{E}( \pm 1 \mathrm{~km}), 02.07 .1994$.

125. $67.044026^{\circ} \mathrm{N} 32.581267^{\circ} \mathrm{E}( \pm 1 \mathrm{~km}), 19.08 .1999$ $-21.08 .1999$

126. $67.086224^{\circ} \mathrm{N} 32.993916^{\circ} \mathrm{E}( \pm 5 \mathrm{~km}), 14.08 .1993$.

127. $67.114409^{\circ} \mathrm{N} 31.553058^{\circ} \mathrm{E}( \pm 1 \mathrm{~km}), 21.07 .1996$

128. $67.145636^{\circ} \mathrm{N} 32.517368^{\circ} \mathrm{E}( \pm 1 \mathrm{~km}), 03.08 .1999$

129. $67.154935^{\circ} \mathrm{N} 33.317163^{\circ} \mathrm{E}( \pm 1 \mathrm{~km}), 17.06 .1994$

130. $67.163783^{\circ} \mathrm{N} 32.498657^{\circ} \mathrm{E}( \pm 1 \mathrm{~km}), 03.07 .2000$.

131. $67.166312^{\circ} \mathrm{N} 33.201071^{\circ} \mathrm{E}( \pm 1 \mathrm{~km}), 08.08 .1993$.

132. $69.205382^{\circ} \mathrm{N} 35.2963257^{\circ} \mathrm{E}( \pm 1 \mathrm{~km}), 12.08 .2003-$ 15.08 .2003

133. $69.2204907^{\circ} \mathrm{N} 35.3758049^{\circ} \mathrm{E}( \pm 1 \mathrm{~km}), 12.08 .2003$.

\section{Primorye Territory}

134. $42.795120^{\circ} \mathrm{N} 131.253779^{\circ} \mathrm{E}( \pm 100 \mathrm{~m}), 25.08 .1996$ $-26.08 .1996$

135. $42.800404^{\circ} \mathrm{N} 131.244494^{\circ} \mathrm{E}( \pm 50 \mathrm{~m}), 15.08 .1996$ -25.08 .1996 .

136. $42.806480^{\circ} \mathrm{N} 131.244648^{\circ} \mathrm{E}( \pm 20 \mathrm{~m})$, a. 19.08 .1996 ; b. $04.09 .2000 ;$ c. 09.2002 .

137. $43.168870^{\circ} \mathrm{N} 132.889915^{\circ} \mathrm{E}( \pm 50 \mathrm{~m})$, a. 23.08 .2000 ; b. 08.2002 .

138. $43.213252^{\circ} \mathrm{N} 132.893210^{\circ} \mathrm{E}(+100 \mathrm{~m}), 30.08 .2000$.

139. $43.631145^{\circ} \mathrm{N} 132.229432^{\circ} \mathrm{E}( \pm 5 \mathrm{~km}), 28.09 .2005$.

$140.43 .730934^{\circ} \mathrm{N} 131.698720^{\circ} \mathrm{E}( \pm 2 \mathrm{~km}), 29.09 .2005$.

\section{Pskov Region}

141. $57.059345^{\circ} \mathrm{N} 28.926145^{\circ} \mathrm{E}( \pm 1 \mathrm{~km}), 22.08 .2014$.

\section{Republic of Karelia}

142. $66.572989^{\circ} \mathrm{N} 32.836019^{\circ} \mathrm{E}( \pm 50 \mathrm{~m})$, a. 01.08 .1992 12.08.1992; b. 01.08.2003 - 10.08.2003.

\section{Ryazan Region}

143. $54.686309^{\circ} \mathrm{N} 40.694984^{\circ} \mathrm{E}( \pm 1 \mathrm{~km}), 20.07 .1997$.

144. $54.686343^{\circ} \mathrm{N} 40.681074^{\circ} \mathrm{E}( \pm 1 \mathrm{~km}), 25.07 .1997$.

145. $54.687702^{\circ} \mathrm{N} 40.561180^{\circ} \mathrm{E}( \pm 1 \mathrm{~km}), 25.07 .1997$.

146. $54.6963567^{\circ} \mathrm{N} 40.8690977^{\circ} \mathrm{E}( \pm 1 \mathrm{~km}), 01.11 .1996$.

147. $54.707761^{\circ} \mathrm{N} 40.862092^{\circ} \mathrm{E}( \pm 100 \mathrm{~m}), 02.12 .1986$

148. $54.708883^{\circ} \mathrm{N} 40.854220^{\circ} \mathrm{E}( \pm 1 \mathrm{~km})$, a. 22.06.1987; b. $24.09 .1990 ;$ c. 10.06 .1996

149. $54.719646^{\circ} \mathrm{N} 40.918454^{\circ} \mathrm{E}( \pm 1 \mathrm{~km}), 24.07 .1997$.

150. $54.721094^{\circ} \mathrm{N} 40.926674^{\circ} \mathrm{E}( \pm 1 \mathrm{~km}), 12.07 .1997$ $-27.07 .1997$

151. $54.721604^{\circ} \mathrm{N} 40.934436^{\circ} \mathrm{E}( \pm 1 \mathrm{~km}), 12.07 .1997$ $-27.07 .1997$

152. $54.725358^{\circ} \mathrm{N} 40.865028^{\circ} \mathrm{E}( \pm 1 \mathrm{~km}), 16.07 .1997$.

153. $54.726700^{\circ} \mathrm{N} 40.857258^{\circ} \mathrm{E}( \pm 1 \mathrm{~km}), 13.07 .1997$ $-17.07 .1997$

154. $54.726700^{\circ} \mathrm{N} 40.857258^{\circ} \mathrm{E}( \pm 2 \mathrm{~km}), 17.07 .1997$.

155. $54.727255^{\circ} \mathrm{N} 40.874405^{\circ} \mathrm{E}( \pm 1 \mathrm{~km}), 24.07 .1997$.

156. $54.729691^{\circ} \mathrm{N} 40.925307^{\circ} \mathrm{E}( \pm 1 \mathrm{~km}), 23.10 .1986$.

157. $54.732286^{\circ} \mathrm{N} 40.963816^{\circ} \mathrm{E}( \pm 1 \mathrm{~km}), 29.05 .1987$.

158. $54.719157^{\circ} \mathrm{N} 40.901206^{\circ} \mathrm{E}( \pm 1 \mathrm{~km}), 24.07 .1997$.

159. $54.719214^{\circ} \mathrm{N} 40.885787^{\circ} \mathrm{E}( \pm 1 \mathrm{~km})$, a. 19.07 .1997 -27.07 .1997 ; b. 19.09.1997.

160. $54.736859^{\circ} \mathrm{N} 40.865661^{\circ} \mathrm{E}( \pm 1 \mathrm{~km}), 13.07 .1997$ $-29.07 .1997$

161. $54.738747^{\circ} \mathrm{N} 40.916205^{\circ} \mathrm{E}( \pm 1 \mathrm{~km}), 24.07 .1997-$ 29.07.1997.

162. $54.741445^{\circ} \mathrm{N} 40.6299734^{\circ} \mathrm{E}( \pm 1 \mathrm{~km}), 24.06 .1987$

163. $54.745987^{\circ} \mathrm{N} 40.848029^{\circ} \mathrm{E}( \pm 1 \mathrm{~km}), 30.07 .1997$.

164. $54.792879^{\circ} \mathrm{N} 40.588680^{\circ} \mathrm{E}( \pm 1 \mathrm{~km}), 22.04 .1997$.

Siem Reap Province (Kingdom of Cambodia)

165. $13.434917^{\circ} \mathrm{N} 103.887783^{\circ} \mathrm{E}( \pm 1 \mathrm{~km}), 20.12 .2014$.

\section{Tver Region}

166. $56.304325^{\circ} \mathrm{N} 34.874414^{\circ} \mathrm{E}( \pm 5 \mathrm{~km})$, a. 03.05 .2015 b. 08.2001 .

167. $56.456333^{\circ} \mathrm{N} 32.960283^{\circ} \mathrm{E}( \pm 5 \mathrm{~m}), 26.04 .2017$

168. $56.672525^{\circ} \mathrm{N} 36.460565^{\circ} \mathrm{E}( \pm 20 \mathrm{~m})$, a. 26.06 .1987 ; b. 20.08.1989; c. 22.04.1990; d. 15.08.199029.08.1990; e. 15.09.1991; f. 08.07.1992; g. 17.06.1993 - 22.06.1993; h. 05.07.1994; i. 22.06.1996-04.07.1996; j. 26.06.1997 03.08.1997; k. 02.06.1998-04.06.1998; 1. 01.07.2001 - 12.07.2001; m. 08.07.2002-07.08.2002; n. $10.07 .2003-10.08 .2003$; o. 10.11.200311.11.2003; p. 22.06.2009 - 28.06.2009; q. 08.1989; r. 08.1990; s. 06.1995.

169. $56.681475^{\circ} \mathrm{N} 36.392279^{\circ} \mathrm{E}( \pm 1 \mathrm{~km})$, a. 07.07.2004; b. 15.07.2006 - 17.07.2006; c. 04.07.2007 07.07.2007.

170. $56.686144^{\circ} \mathrm{N} 36.568391^{\circ} \mathrm{E}( \pm 1 \mathrm{~km}), 11.07 .2007$.

171. $56.701150^{\circ} \mathrm{N} 36.418700^{\circ} \mathrm{E}( \pm 500 \mathrm{~m}), 10.07 .2003$ $-11.07 .2003$

172. $56.804377^{\circ} \mathrm{N} 37.057179^{\circ} \mathrm{E}( \pm 100 \mathrm{~m}), 07.01 .1993$.

173. $57.584893^{\circ} \mathrm{N} 36.215374^{\circ} \mathrm{E}( \pm 500 \mathrm{~m}), 19.08 .1993$.

\section{Tyumen Region}

174. $56.956338^{\circ} \mathrm{N} 65.763138^{\circ} \mathrm{E}( \pm 5 \mathrm{~km})$, a. 05.06 .2008 -08.06 .2008 ; b. 2008; c. 07.2009

175. $57.075664^{\circ} \mathrm{N} 65.077175^{\circ} \mathrm{E}( \pm 5 \mathrm{~km})$, a. 10.06 .2001 ; b. $20.05 .2004-03.06 .2004$.

176. $57.106214^{\circ} \mathrm{N} 65.067743^{\circ} \mathrm{E}( \pm 5 \mathrm{~km})$, a. 15.05 .1996 b. 12.05 .1997 .

\section{Vladimir Region}

177. $55.881672^{\circ} \mathrm{N} 39.458536^{\circ} \mathrm{E}( \pm 1 \mathrm{~km}), 27.08 .1992$.

\section{Yaroslavl Region}

178. $57.032646^{\circ} \mathrm{N} 38.644730^{\circ} \mathrm{E}( \pm 1 \mathrm{~km}), 25.08 .1993$.

\section{Unknown area}

179. Collection locality and collection date are not specified. 


\section{RESULTS}

The species list is annotated with boldface numeric codes of collection locations, alphabetic indices of collection dates (see the list of collecting sites) and herbarium numbers in the Myxomycetes collection of CYN in parentheses. Myxomycete nomenclature follows Lado (2005-2020), except for some varieties and subspecies, which are not accepted in this information system, and Stemonitis lignicola Nann.-Bremek., which we consider as a separate species.

Amaurochaete atra (Alb. et Schwein.) Rostaf. - 168j (662). New for Tver Region.

Arcyodes incarnata (Alb. et Schwein.) O.F. Cook - 20 (13881); 94 f (189); 110o (1479-1).

New for Khabarovsk Territory.

Arcyria affinis Rostaf. - 5 (1571); 7 (491); 23 (762); 27 (355); 29b (231); 35c (35); 78b (330-1); 78g (282-1); 78i (417); 78j (531); 78n (516); 78o (715); 78q (1229); 78t (28, 255); 83a (760); 89h (1263, 1589); 94i (851); 96a (450); 105b (503, 506, 507); 107d (376); 109 (375, 388, 389); 110e (1322); 110h (838-1); 110k (1430); 110m (1543); 110o (1467); 110s (1114); 121 (819); 132 (804); 135 (421); 136a (382, 393); 166b (673); 168i (442); 174 a $(936,937-1,983)$.

New for Tyumen and Murmansk Regions.

Arcyria cinerea (Bull.) Pers. - 16c (1086, 1092, 1107-1); 43 (364); 69a (1560); 78g (314, 327); 78x (1514-1); 85e (1356); 88 (1277); 94d (114); 105b (504-1); 109 (422); 110e (12872, 1335); 110i (885); 110k (1424); 110m (1544); 110o (1468, 1478); 117 (1527-1, 1637); 125 (485); 137a (1034); 137b (1014); 168m (774); 169c (1039); 174a (938, 984).

New for Irkutsk and Tyumen Regions.

Arcyria denudata (L.) Wettst. - 27 (357); 67c (148); 69a (1557); 78g (286); 88 (1218); 89i (628); 94d (108); 110c (617); 110m (1545); 137b (1007); 149 (557); 169c (1078); 174b (1046). New for Tyumen Region.

Arcyria ferruginea Saut. - 40 (342); 45d (38); 49 (494); 51 (433); 53b (176); 55a (138); 57 (1189); 77a (32); 78g (306); $78 \mathrm{i}$ (386); 93 (331); 102 (79); 105b (500); 107a (87); 110a (34); $110 \mathrm{e}(1137,1146,1288,1289-1,1311) ; 110 \mathrm{~h}(833,838-2)$.

Arcyria helvetica (Meyl.) H. Neubert, Nowotny et K. Baumann (Fig. 2A, B) - 4 (489); 110e (1135).

New for Crimean Peninsula, Moscow and Moscow Region.

Arcyria imperialis (G. Lister) Q. Wang et Yu Li - 16c (1089-1, 1090); 67a (40); 67e (39); 89h (1274-1); 137b (1002); 139 (1511).

New for Irkutsk Region, Moscow and Moscow Region.

Arcyria incarnata (Pers. ex J.F. Gmel.) Pers. - 16b (1396-1); 29b (217, 218); 45e (41); 56 (206); 67b (133); 68 (996); 78g (282-2); 85a (121); 89d (743, 754, 755); 89h (1258, 1590-1); 94i (855); 110h (1402); 110o (1482); 117 (1519); 132 (816); 143 (563); 159a (556); $161(571,572) ; 168 b$ (33); 168m (624); 174a (937-2); 174b (1042).

New for Irkutsk, Tyumen, and Murmansk Regions.

Arcyria obvelata (Oeder) Onsberg - 7 (492); 77a (36); 78g (303); 78j (522, 523, 692); 78o (716); 78t (251); 102 (78-1); 107d (414); 110a (37); 113a (360); 117 (1521, 1524); 123 (405); 132 (801); 141 (1437, 1598-1); 142b (725); 151 (566); 153 (568); 160 (567); 165 (1520); 166b (674); 168h (441); 168j (997); 168k (533); 1681 (690); 168p (1445-1); 169b (1320); 169c (1085); 171 (794, 797); 174a (964); 174b (1044).

Arcyria occidentalis (T. Macbr.) G. Lister, in Lister (Fig. 2C) - 16b (1384); 20 (1393, 1585-1); 78g (308); 78x (289); 110e $(1286,1328)$

New for Khabarovsk Territory and Irkutsk Region.

Arcyria oerstedii Rostaf. - 78b (330-2); 78j (515); 78o (717); 89h (1271); 110e (1318); 110j (1020); 112a (369); 175b (892).

New for Tyumen Region.

Arcyria pomiformis (Leers) Rostaf. - 29b (241); 69a (1566); 78g (301); 89d (751); 89h (915-1, 922); 105b (504-2); 110h (832); 1101 (1415); 168j (998); 174a (939, 985); 174b (1037, 1213).
Arcyria stipata (Schwein.) Lister - 65a (629); 69a (1562); 78k (526); 94d (109); 109 (378); $110 f$ (1284).

Badhamia affinis Rostaf. - 16b (1396-2); 94f (188); 110f (1122); 110t (883).

New for Irkutsk Region.

Badhamia capsulifera (Bull.) Berk. (Fig. 2D) - 54 (243-1); 55a (139).

Badhamia foliicola Lister - 19 (1226); 53b (168); 79 (415); 105a (413); 159a (573).

New for Kaluga and Ryazan Regions.

Badhamia lilacina (Fr.) Rostaf. - 78g (267); 781 (519, 710); 142a $(173,174)$.

New for Republic of Karelia.

Badhamia macrocarpa (Ces.) Rostaf. - 29e (1615); 55b (1419); 64a (865, 871); 96c (582, 583); 97 (83); 110m (1541, 1547); 110p (1630); 117 (1522); 168e (335).

Badhamia utricularis (Bull.) Berk. - 45b (84); 53b (167); 77c (655); 81b (864); 100b (1368); 110h (842); 110i (884); 110q (652); 111 (1507).

Ceratiomyxa fruticulosa (O.F. Müll.) T. Macbr. - 69a (1564); 89h (915-2); 110o (1460, 1465, 1487-1); 141 (1598-2); 168p (14381, 1440, 1441, 1445-2); 169a (1597); 174a (933); 174b (1227).

New for Tyumen Region.

Ceratiomyxa fruticulosa var. flexuosa (Lister) G. Lister, in Lister - 16c (1145); 78g (268); 86 (850-1); 89d (734); 109 (406); 110k (1422); 168g (202); 168j (469); 168k (537); 1681 (689); 168m (631); 168n (775-1); 169c (1053).

New for Irkutsk Region.

Ceratiomyxa fruticulosa var. porioides (Alb. et Schwein.) G. Lister, in Lister - 29b (221, 222); 78g (302); 78q (1183); 88 (1252).

Clastoderma debaryanum A. Blytt - 20 (1389, 1512); 139 (1600).

Collaria arcyrionema (Rostaf.) Nann.-Bremek. ex Lado 29b (220); 40 (340, 343); 44 (366); 78g (275, 315, 322); 89c

(848); 110e (1143-1); 113b (445); 117 (1417); 125 (478); 163 (550); 168i (444); 168m (635); 169c (1066); 174a (951, 953, 960, 963, 969); 174b (1041, 1236).

New for Tyumen Region.

Comatricha ellae Härk. - 110o (1487-2); 132 (807).

Comatricha laxa Rostaf. - 29b (213, 214).

Comatricha nigra (Pers. ex J.F. Gmel.) J. Schröt., in Cohn - 11 (656); 29a (224, 225); 29b (223); 53a (136); 60 (69); 67a (63); 78x (332); 85e (1029); 94c (66); 97 (68); 100d (593); 109 (420, 424); 110a (64); 110e (1310-1); 110g (907); 110h (1381); 110k (1427); 110m (1537-1); 110o (1457, 1461); 112a (368); 117 (1418, 1436); 168p (1450); 170 (1171); 174b (1248).

Comatricha pulchella (C. Bab.) Rostaf. - 111 (1501-1); 142b (724); 168j (663, 664); 174a (949).

Comatricha cf. reticulospora Ing et P.C. Holland - 110-e (1300); 132 (810).

New for Moscow, Moscow and Murmansk Regions.

Craterium aureum (Schumach.) Rostaf. - 78-g (296).

Craterium leucocephalum (Pers. ex J.F. Gmel.) Ditmar, in Sturm - 78g (278); 85a (112); 89d (740); 89g (1243-1, 1246, 1256); 89h (879, 921, 1264); 132 (805).

New for Murmansk Region.

Craterium minutum (Leers) Fr. - 78g (266, 316); 110e (1125, 1350); 174a (966); 174b (1235).

New for Tyumen Region.

Craterium obovatum Peck - 16c (1094, 1108). New for Irkutsk Region.

Cribraria argillacea (Pers. ex J.F. Gmel.) Pers. - 16c (1095); 29b (227); 44 (367); 78g (290, 304); 78q (1270); 89d (737, 738, 739-1); 89h (1299); 110o (1454); 152 (560); 162 (599); 174a (955, 981); 174b (1045).

New for Irkutsk, Ryazan, and Tyumen Regions.

Cribraria aurantiaca Schrad. - 29b (226); 78g (288); 174a (943-1). 


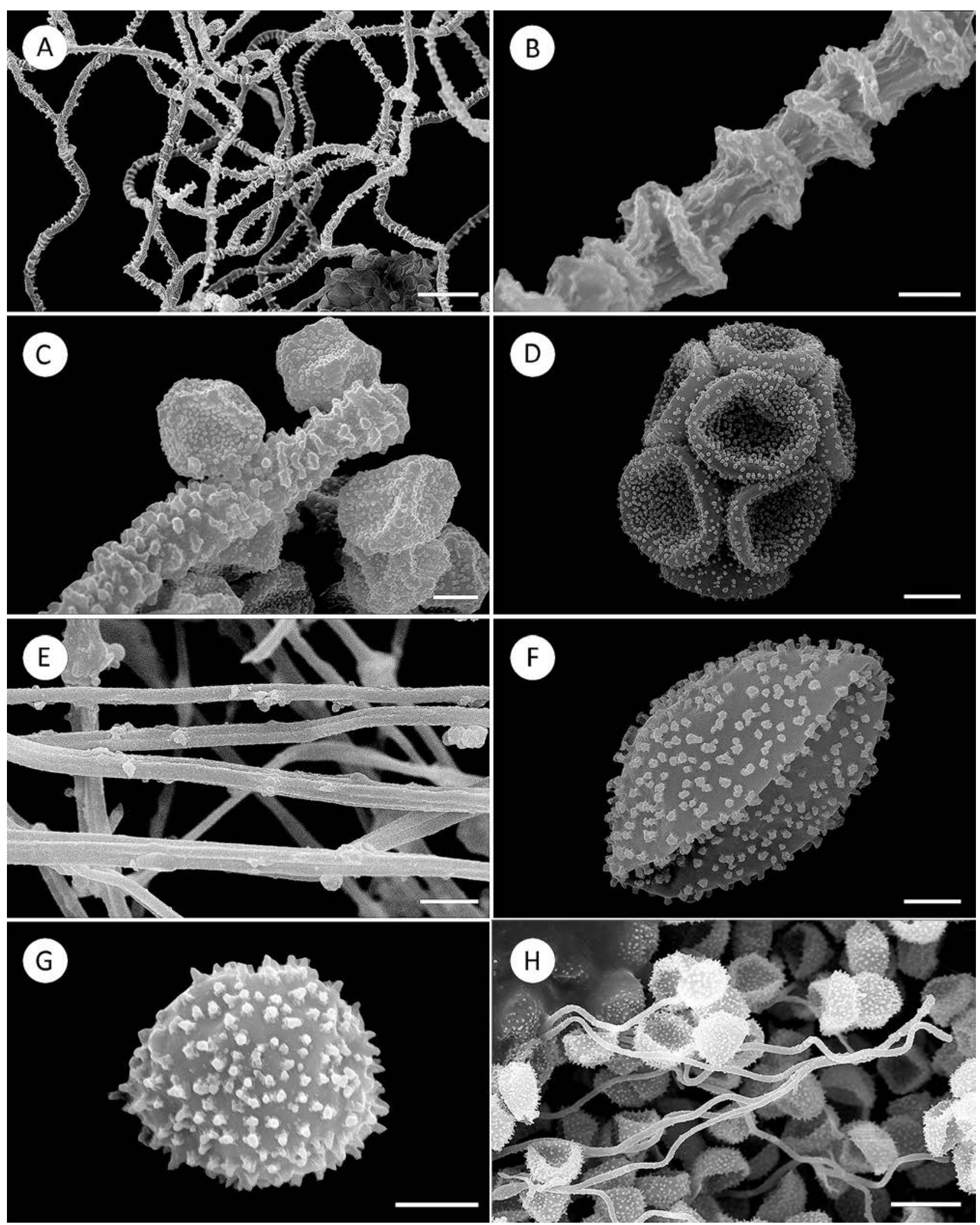

Figure 2 SEM photographs. A-B - Arcyria helvetica (Meyl.) H. Neubert, Nowotny et K. Baumann (489): A - capillitium network and fragment of peridium, B - ornamentation of thread of capillitium; C - Arcyria occidentalis (T. Macbr.) G. Lister (289), fragment of capillitium and spores; D - Badhamia capsulifera (Bull.) Berk. (243), cluster of spores; E-F - Diderma spumarioides (Fr. et Palmquist) Fr. (243): E - fragment of capillitium, F - spore; G-H - Diderma cinereum Morgan (191): G - spore, H - spores and capillitium. Scale bars: A - $30 \mu \mathrm{m}, \mathrm{B}, \mathrm{C}, \mathrm{G}-3 \mu \mathrm{m}$; $\mathrm{D}, \mathrm{E}-5 \mu \mathrm{m} ; \mathrm{F}-2 \mu \mathrm{m} ; \mathrm{H}-10 \mu \mathrm{m}$ 
Cribraria cancellata (Batsch) Nann.-Bremek. - 16c (1100, 1109); 29b (190); 39 (348); 78g (293); 78o (711); 78q (1162, 1165); 78t (254); 78x (1514-2); 86 (850-2); 89c (849); 89h (1267); 110k (1428); 110o (1462, 1463, 1475); 136c (1015); 137b (1012); 148a (596); 151 (564, 565); 168a (660); 1681 (685); 168m (605); 168n (787); 174a (935); 174b (1047).

New for Irkutsk Region.

Cribraria intricata Schrad. - 174a (978). New for Tyumen Region.

Cribraria languescens $\mathrm{Rex}-100 \mathrm{~d}$ (588).

Cribraria macrocarpa Schrad. - 89d (739-2); 174a (958). New for Tyumen Region.

Cribraria microcarpa (Schrad.) Pers. - 69a (1554, 1558); 110h (846); 151 (562); 169c (1084); 174b (1160). New for Ryazan and Tyumen Regions.

Cribraria oregana H.C. Gilbert, in Peck et Gilbert - 78y (461); 137b (1009); 174a (974).

New for Moscow, Moscow and Tyumen Regions.

Cribraria piriformis Schrad. - 78y (430); 110o (1471); 168m (638); 168n (778).

Cribraria purpurea Schrad. - 35b (1); 110m (1552, 1587); 111 (1500).

Cribraria rufa (Roth) Rostaf. - 85a (118); 89a (640); 89e (1233, 1255); 89h (920); 110h (837, 1376); 110s (1115); 111 (1493, 1499-1); 169c (1063, 1082).

Cribraria tenella Schrad. - 35d (99); 161 (561).

Cribraria violacea Rex - 69b (1210).

Cribraria vulgaris Schrad. - 72 (1211); 132 (811); 174a (943-2). New for Tyumen Region.

Diachea leucopodia (Bull.) Rostaf. - 78a (294); 88 (1215).

Dictydiaethalium plumbeum (Schumach.) Rostaf., in Lister - 81a (867).

Diderma cinereum Morgan (Fig. 2G, H) - 29b (191).

Diderma effusum (Schwein.) Morgan - 78g (323); 137b (989).

Diderma radiatum (L.) Morgan - 16c (1154); 35d (94); 67d (927); 94b (30); 110c (644); 110e (1319); 129 (337); 170 (1173); $174 a$ (961).

New for Irkutsk Region.

Diderma spumarioides (Fr. et Palmquist) Fr. (Fig. 2E, F) 54 (243-2); 140 (1398).

Didymium anellus Morgan - 78o (707, 708).

Didymium clavus (Alb. et Schwein.) Rabenh. - 78g (283).

Didymium crustaceum Fr. - 78g (276).

Didymium difforme (Pers.) Gray - 69a (1556); 82 (1195); 83b (1197).

Didymium iridis (Ditmar) Fr. - 89g (1244).

Didymium megalosporum Berk. et M.A. Curtis, in Berkeley (Fig. 3A-C) - 89d (745-1).

New for Moscow and Moscow Region. D. megalosporum is superficially similar to $D$. ovoideum, but has a compact flat columella (Fig. 3A). D. eximium Peck. is different due to stellate peridial crystals which are smaller than spores. When it comes to D. megalosporum, they are always larger than the spores. In Russia, this species was described only for the Volgograd Region (Zemlianskaia 2003, Novozhilov et al. 2003, Novozhilov 2005).

Didymium melanospermum (Pers.) T. Macbr. - 56 (208); 75b (667); 78g (284); 78i (412, 432); 89d (744); 89g (1247); 105b (501); 106 (76); 107a (101, 117); $107 \mathrm{~b}$ (196); 108 (145); 110e (1342); 146 (595); 168d (24); 1680 (1240); 171 (793); 178 (194). New for Ryazan and Yaroslavl Regions.

Didymium minus (Lister) Morgan - 50 (1518); 66 (1222); 78o (703, 704, 706); 88 (1217); 89g (1243-2); 94f (186); 110e (1312); 110k (1421); 117 (1434, 1532); 151 (578). New for Ryazan Region.

Didymium nigripes (Link) Fr. - 78t (247); 89i (641); 137b (986).

Didymium ovoideum Nann.-Bremek. (Fig. G-I) - 78g (277). New for Moscow and Moscow Region. This species is distinguished by an oval shape of sporangia with long, thin, reddishbrown stalk, large clavate columella that reaches the centre of sporangium (Fig. 3G), and evenly warted spores (Fig. 3I). It was previously reported only from Altay Republic (Novozhilov 2005, Novozhilov et al. 2010). Like D. megalosporum D. ovoideum is very similar to D. iridis (Ditmar) Fr. According to Poulain et al. (2011a): "D. iridis, which belongs to this group, is a poorly defined species with several interpretations". Apparently, it is a group of similar morphospecies on long, limeless, semi-translucent stalks.

Didymium squamulosum (Alb. et Schwein.) Fr. et Palmquist 14 (1602); 46b (127); 59 (1200); 69a (1555); 78g (310); 82 (1194 1196); 83b (1198); 88 (1232); 89b (642); 138 (1032); 174a (982). New for Tyumen Region.

Enerthenema papillatum (Pers.) Rostaf. - 29b (216, 240); 78g (273); 78s (253); 107b (195); 110e (1310-2); 132 (809); 133 (822); 161 (548); 168j (661); 168n (777); 171 (792); 174a (959, 971); 174b (1237).

Fuligo cinerea (Schwein.) Morgan - 160 (551). New for Ryazan Region.

Fuligo intermedia T. Macbr. (Fig. 3D-F) - 3a (1516). New for Russia (Astrakhan Region).

Description. Sporophores are aethalia, often forming large groups, pulvinate, $0.5-3 \mathrm{~cm}$ in diam., up to $1 \mathrm{~cm}$ height, occasionally up to $6 \mathrm{~cm}$ or more, covered with crust, spongy, yellowish-grey cortex, which detaches from the spore mass in the form of flaky fragments (Fig. 3F). When cortex breaks up and spores disperse, sporophore turns into a mass of interweaved, small, light-coriaceous films which form an abundant pseudocapillitium. Hypothallus firm and spongy; it remains for a long time after aethalium is broken. Capillitium consists of large angular lime nodes connected by hyaline threads and united into a net with few free ends (Fig. 3E). Occasionally, lime knots gather in the centre and form a loose pseudocolumella. Spores black in mass; light-brown in transmitted light, globose, $10-12 \mu \mathrm{m}$ in diam., minutely warted (Fig. 3D). Plasmodium white by color (according to Martin \& Alexopoulos 1969).

Notes. Fuligo intermedia is very similar to F. septica var. candida, but differs by large spores $(10-12 \mu \mathrm{m}$ vs. $7-9 \mu \mathrm{m}$ respectively). F. candida differs by oval spores, smaller size of sporophore, and a poorly developed hypothallus. In addition, F. cinerea has a thin cortex firmly attached to a sporophore that never falls off as white flakes. However, sometimes F. intermedia has an underdeveloped cortex (see Poulain et al. 2011b, Fig. 179), but individual sporangia still preserve peridium. In this case, spore diameter and capillitium structure are the only reliable diagnostic features of this species.

Fuligo leviderma $\mathrm{H}$. Neubert, Nowotny et K. Baumann 16c (1087); 53b (166); 67b (134); 76 (463); 78g (305); 81a (858); 85a (122); 89f (701); 94b (6); 94e (157); 94h (462); 96a (449); 100b (451); 101 (17); 102 (16); $110 f$ (1638); 110g (913); $110 \mathrm{~h}$ (834); 110m (1548); 110p (1626); 168c (18); 169c (1062); 170 (1170); 176a (767). New for Irkutsk Region.

Fuligo luteonitens L.G. Krieglst. et Nowotny, in Neubert, Nowotny et Baumann - 35d (93). New for Moscow and Moscow Region.

Fuligo septica (L.) F.H. Wigg. - 27 (354); 29b (228); 37 (3492); 45c (179); 64b (870); 78g (300-2); 78j (518); 78k (464); 78q (1163, 1575); 78s (252); 87 (1576); 92 (1509); 103 (181); 109 (401, 416); 126 (244); 130 (800); 141 (1435); $142 b$ (720); 144 (553); 151 (466); 157 (603); 168p (1438-2); 174a (1432).

Fuligo septica var. candida (Pers.) R.E. Fr. - 37 (349-1); 38 (352); 78g (274, 300-1); 78j (528); 85g (857); 107b (242); 109 (384); 110n (1433); 125 (477, 479, 480, 481); 153 (552); 160 (554); $168 \mathbf{i}(856) ; 174 b$ (1234).

Fuligo septica var. rufa (Pers.) Lázaro Ibiza - 153 (555).

Hemitrichia abietina (Wigand) G. Lister, in Lister-174a (940). New for Tyumen Region.

Hemitrichia calyculata (Speg.) M.L. Farr - 64a (861-1); 71 (877); 78d (153); 78g (270, 320, 333); 78i (455-1); 78m (512); 78u (263); 85e (1026); 94d (31); 102 (78-2); 110e (1340); 110g (910); 110h (1374); 110m (1536); 110s (1111); 111 (1501-2, 1508); 137b (1008); 147 (600); 176b (669).

New for Ryazan and Tyumen Regions.

Hemitrichia clavata (Pers.) Rostaf., in Fuckel - 16c (1091, 1093, 1096, 1097, 1107-2); 20 (1513-1, 1582); 29e (1603); 47 (1534); 57 (1209); 78d (150); 78g (295); 78i (455-2); 78y (460); 85e (1360, 1361, 1379); 85f (1515); 89h (1261, 1274-2); 

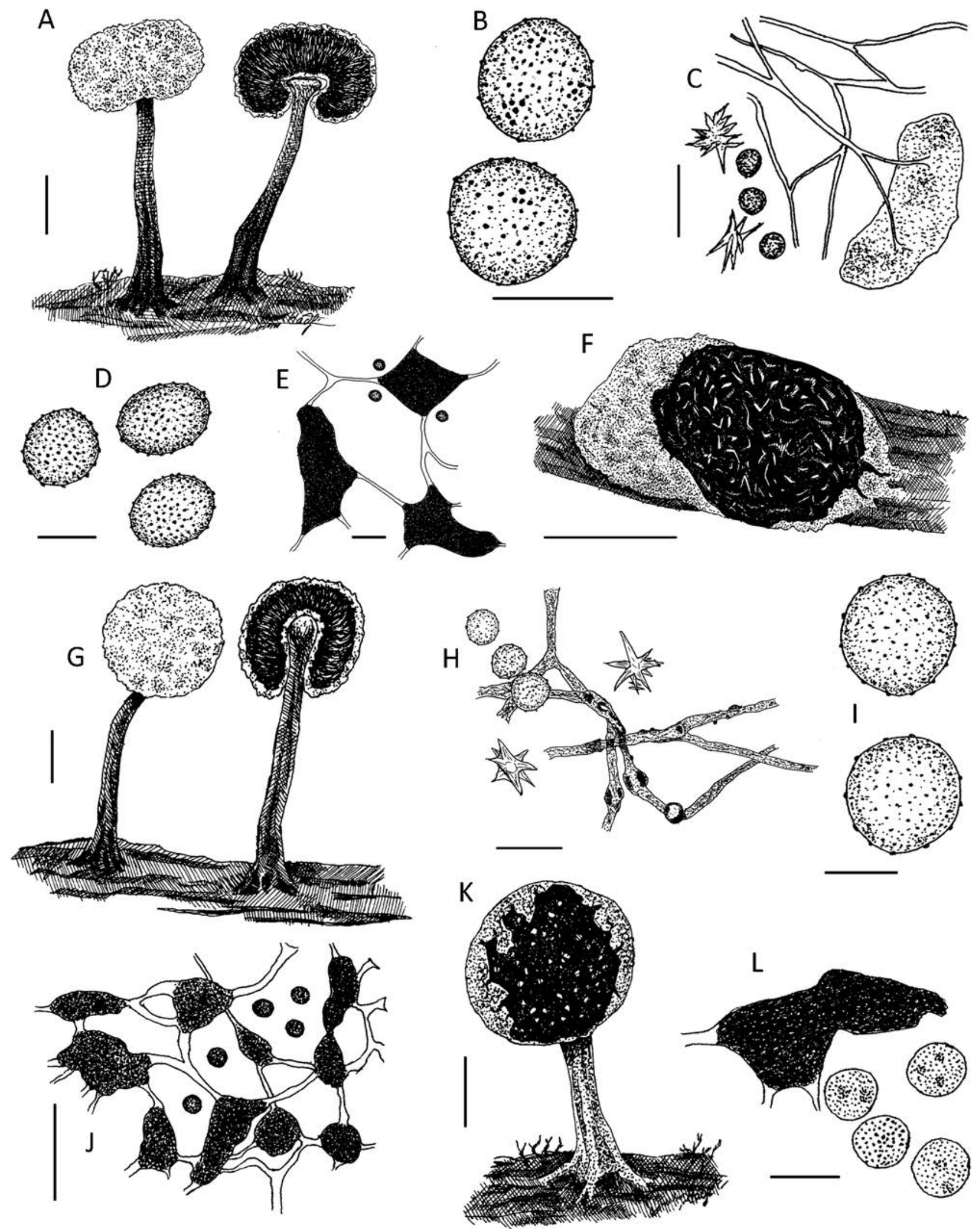

Figure 3 Drawings. A-C - Didymim megalosporum Berk. et M.A. Curtis (collection number 745), A - two sporophores with flat columella, B - spores as seen under oil immersion, C - capillitium, spores and stellate lime crystals; D-F - Fuligo intermedia T. Macbr. (1516), D - spores, E - capillitium and spores, F - aethalium; G-I - Didymium ovoideum Nann.-Bremek. (277), G - two sporophores with clavate columella, H - capillitium, spores and stellate lime crystals, I - spores; J-L - Physarum murinum Lister (576), J - capillitium and spores, K - sporophore, L - spores and lime nodule. Scale bars: A - $0.3 \mathrm{~mm} ; \mathrm{B}, \mathrm{E}-25 \mu \mathrm{m} ; \mathrm{C}, \mathrm{D}, \mathrm{L}-10 \mu \mathrm{m} ; \mathrm{F}-1 \mathrm{~mm} ; \mathrm{G}-0.2 \mathrm{~mm} ; \mathrm{H}-20 \mu \mathrm{m}$; I - $5 \mu \mathrm{m}$; J $-50 \mu \mathrm{m}$; $\mathrm{K}-0.2 \mathrm{~mm}$ 
110c $(616,700) ; 110$ e $(1293,1341-1) ; 110 f(1130,1307) ; 110$ h $(829,1369,1380,1403-1) ; 110 p(1616,1632) ; 111(1501-3) ; 117$ (1533); 164 (602); 170 (1175); 174a (970); 175b (888-1, 896).

New for Irkutsk Region.

Hemitrichia serpula (Scop.) Rostaf. ex Lister - 16c (1106); 29e (1608, 1609); 78h (456); 85e (1027); 89d (753); 110c (620); 110e (1324, 1329); 110g (909); 110h (840); 110p (1629); 111 (1506-1, 1593-1); 137b (1000); 140 (1399); 166a (1407); 169c (1069, 1072); 175b (895).

New for Irkutsk and Tyumen Regions.

Lamproderma arcyrioides (Sommerf.) Rostaf. - 174b (1253). New for Tyumen Region.

Lamproderma columbinum (Pers.) Rostaf., in Fuckel - 77d (131); 85a (119); 89g (1245-1); 89h (1260); 110h (843); 110m (1537-2); 111 (1491, 1492, 1494, 1495-1, 1496, 1498-1).

Lamproderma scintillans (Berk. et Broome) Morgan - 61 (1212); 69a (1561); 110e (1152).

Leocarpus fragilis (Dicks.) Rostaf. - 6 (1296); 48 (1017); 55a (140); 56 (207, 209); 78g (272); 781 (509); 780 (709); 78t (77, 249); 80 (329); 89d (742); 89h (924); 107a (14, 89); 107b (197); 110e (1127); 110o (1486); 146 (468); 168d (15); 168n (788); 178 (193).

Lepidoderma tigrinum (Schrad.) Rostaf., in Fuckel - 35d (100); 94d (105); 111 (1489, 1498-2, 1501-4).

Licea minima Fr. - 64d (1230); 78q (1177, 1179); 174a (968). Licea operculata (Wingate) G.W. Martin - 64e (1205, 1220). Lindbladia tubulina Fr. - 168p (1451).

Lycogala epidendrum (L.) Fr. -9 (584); 12 (606); 13 (773); 16b (1396-3); 16c (1110); 16d (931); 20 (1394); 29b (187); 32 (67); 45a (11); 45e (7, 12-1); 46a (9); 53b (70); 55a (156); 65b (1035); 76 (988); 77a (8); 78g (299); 78j (525); 78q (1161); 84 (395); 85c (882); 85e (1023, 1359); 88 (1221); 89d (736); 89f (702); 89h (1272); 94a (10); 94d (13-1); 105b (502); 107a (92); 110c (614); 110e (1118); 110f (1121, 1301); 110g (911); 110h (839, 1377); 110k (1423-1); 1101 (1410); 110m (1539, 1551); 110o (1464); 110p (1634); 110s (1113); 113b (362); 121 (817); 125 (475); 127 (409); 132 (813); 133 (823); 136c (1005); 139 (1601); 142a (172); 142b (726); 159a (580); 166b (676); 168k (535); 1681 (686); 168m (623); 168p (1446); 169b (1176); 169c (1051); 174a (934, 956, 957); 174c (1596); 175a (627); 175b (898); 177 (142). Lycogala exiguum Morgan - 78g (292); 78q (1164); 89e (1257); 110k (1423-2); 110p (1633); 132 (802); 136a (403); 169c (1079); 175b (893).

New for Murmansk Region.

Macbrideola cornea (G. Lister et Cran) Alexop. - 62 (1298). Metatrichia floriformis (Schwein.) Nann.-Bremek. - 16c (1088); 29e (1611); 77d (129); 94d (106, 107); 110e (1336).

New for Irkutsk Region.

Metatrichia vesparia (Batsch) Nann.-Bremek. ex G.W. Martin et Alexop. - 16b (1395); 16d (929); 20 (1513-2); 29e (1607, 1610); 30 (604, 731); 33 (431); 35a (44); 35d (98); 39 (350); 45e (12-2); 46b (128); 47 (1535); 64a (862-1); 69a (1559); 78d (159); 78g (265, 334); 78i (452); 78j (517, 530); 78k (521); 81b (863); 85a (120); 85b (696); 85e (1352); 89h (1275); 100a (185); 104 (373); 108 (144); 110c (619); 110e (1308); 110f (1309, 1351-1); 110g (903); 110h (831); 110m (1542); 110p (1619, 1622); 110s (1124); 113b (359); 115 (158); 137b (1013); 156 (601); 166b (675); 168m (622-1, 639-1); 169c (1054, 1064); 174a (941, 9612); 174b (1040); 175b (888-2); 176a (768); 176b (671).

New for Irkutsk Region.

Mucilago crustacea F.H. Wigg. - 5 (1567, 1568, 1570, 1572, 1573, 1579); 29b (229); 56 (205); 78g (328); 78-t (260); 122 (486); 137b (987, 991, 994); 149 (577); 151 (570).

Perichaena chrysosperma (Curr.) Lister - 2 (1282, 1283); 85e (1028, 1354).

Perichaena corticalis (Batsch) Rostaf. - 22 (765); 25 (766); 37 (346); 58 (1280); 78f (457); 78g (311); 85e (1353); $94 \mathbf{i}(853) ; 110 \mathrm{e}$ (1126, 1302); 110g (925); 1101 (1406); 168g (201); 173 (198).

Perichaena corticalis var. liceoides (Rostaf.) G. Lister, in Lister - 110b (182).

Perichaena depressa Lib. - 59 (1199); 70 (1202); 78p (1186, 1574); 83b (1204).
Physarum album (Bull.) Chevall. - 20 (1388-2); 29b (234, 235); 35d (104); 64a (859); 69a (1563); 77a (20); 78g (280-1); 78k (508); 78q (1166, 1182); 81a (866); 83a (758); 85e (874); 89d (741, 745-2); 89g (1245-2); 89h (916, 1266, 1590-2); 90 (1219); 100d (589); 105b (505); 107d (383); 109 (426); 110c (647, 648); 110e (1290, 1291, 1314, 1317, 1344); 110f (1140); 110h (828, 844, 1364, 1372, 1401); 1101 (1412); 110m (1588); 110o (1456, 1466, 1469); 110p (1625, 1627); 112b (370); 114 (385); 120 (798); 136a (404); 137b (1004); 160 (579); 168d (21, 26, 27, 65); 168n (775-2, 779, 786); 168o (1239, 1241); 171 (795); 174a (945-1, 946); 174b (1038, 1156, 1157, 1158, 1159); 175b (897). New for Ryazan Region.

Physarum auriscalpium Cooke (Fig. 4A) - 159b (574). New for Ryazan Region.

Physarum bivalve Pers. (Fig. 4B, C) - 75a (496); 78g (307); 78o (698); 89h (1262).

Physarum cinereum (Batsch) Pers. - 57 (1208); 59 (1201-1); 78d (151); 78g (318); 78o (705); 78q (1180); 78t (246); 88 (1279); 100d (590); 110o (1484); 168n (928); 171 (790, 791).

Physarum confertum T. Macbr. - 171 (796). New for Tver Region.

Physarum contextum (Pers.) Pers. - 110h (1373).

Physarum diderma Rostaf. - 84 (435); 101 (23).

Physarum didermoides (Pers.) Rostaf. - 81a (868).

Physarum flavicomum Berk. - 29b $(237,238)$.

Physarum globuliferum (Bull.) Pers. - 154 (558); 168n (785); 174a $(948,979)$. New for Tyumen Region.

Physarum leucophaeum Fr. et Palmquist - 16b (1382); 20 (1585-2); 45a (25); 75a (495); 76 (693, 694); 78v (427); 85e (1030, 1031); 89g (1242); 94d (110); $94 \mathbf{i}(854) ; 95$ (374); 110c (649); 110e (1289-2, 1331, 1345); 110f (1119); 110g (906); 110o (1479-2); 110q (653); 119 (799); 121 (818); 169c (1056, 1065, 1070, 1081); 175b (891).

New for Irkutsk and Tyumen Region.

Physarum leucopus Link - 20 (1387); 21 (1193); 29b (230); 35d (96); 59 (1201-2); 73a (875); 85e (873); 89h (881); 110c (646); 110e (1132); 110h (1370); 169c (1061, 1067, 1149); 174a (961-3). New for Khabarovsk Territory and Tyumen Region.

Physarum melleum (Berk. et Broome) Massee - 21 (1192).

Physarum murinum Lister (Fig. 3J-L) - 153 (576).

New for Ryazan Region. This species is distinguished by globose sporocarps on long stalks (Fig. 3K), dense capillitium with numerous junctions with small, round, brown nodes connected by thin translucent tubes (Fig. 3J). Capillitium remains in the form of sporangium after spore dispersal. This species is closely related to Physarum globuliferum, but differs by pale brown, not white lime nodes. This is the first record of Physarum murinum in Russia confirmed by a herbarium specimen. Previously it was described only for Moscow region (Sizova \& Titova 1985, Barsukova \& Dunaev 1997, Novozhilov 2005), but there were no specimens to confirm identification.

Physarum mutabile (Rostaf.) G. Lister, in Lister - 78g (324).

Physarum nitens (Lister) Ing (Fig. 5D-F) - 109 (400).

New for Moscow and Moscow Region. This species is distinguished by long plasmodiocarps, slightly curved, with irregular shape, sometimes heaped (Fig. 5D), yellow, with large nodes of capillitium and small light spores ( $7-9 \mu \mathrm{m}$ in diam.) uniformly covered with small warts. It was previously described as P. virescens var. nitens Lister. Typical form of P. virescens differs by pseudoaethalia composed of heaped sporangia, which aggregate in a compact structure. $P$. luteolum is distinguished from $P$. nitens by light-yellow lime on top of sporangia and bigger spores $(8.5-$ $11 \mu \mathrm{m})$. In addition, P. luteolum is characterised by clustered but not heaped sporangia. In Russia, it was reported only for Altai by N.N. Lavrov (1929).

Physarum notabile T. Macbr. - 20 (1391); 45e (19); 53b

(170); 107a (80); 174a (973). New for Khabarovsk Territory and Tyumen Region.

Physarum penetrale Rex (Fig. 5G) - 168d (22).

This unusual species is characterized by thin, membranous, slightly silvery iridescent peridium, long, thin stalks and long columella that penetrates the whole sporangium (Fig. 5G), solid capillitium network formed by thin hyaline tubes with compact 


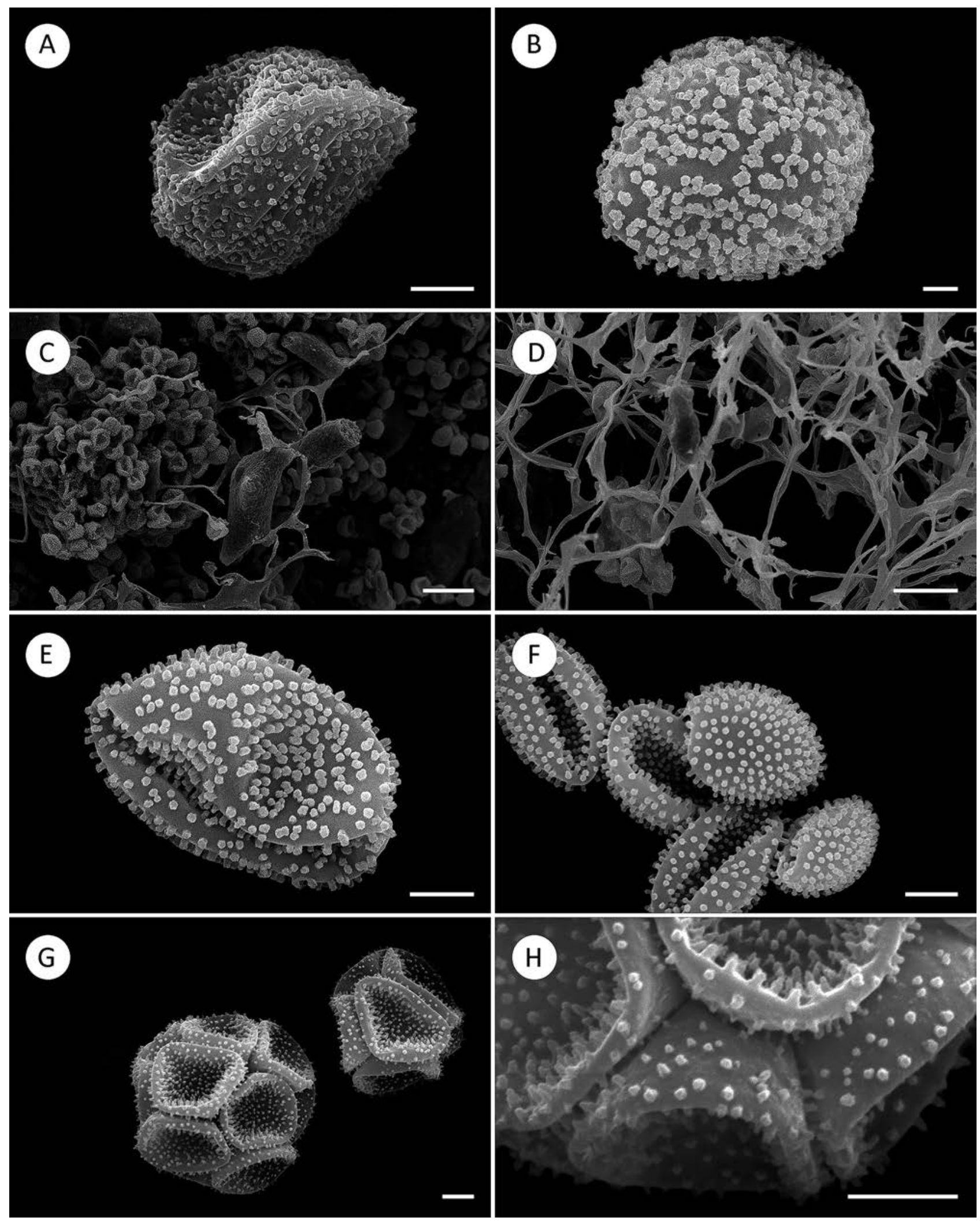

Figure 4 SEM photographs. A - Physarum auriscalpium Cooke (574), spore; B-C - Physarum bivalve Pers. (37): B - spore, C - fragment of capillitium with large, rounded nodules and spores; D-E - Physarum flavicomum Berk. (237): D - fragment of capillitium and spores, E - spores; F - Stemonaria irregularis (Rex) Nann.-Bremek., R. Sharma et Y. Yamam. (1612), spores; G-H - Stemonitis uvifera T. Macbr. (462): G - two clusters of spores, $\mathrm{H}$ - fragment of spore cluster, spores warted on the outer surface, nearly smooth elsewhere. Scale bars: A, E - $2 \mu \mathrm{m} ; \mathrm{B}-$ $1 \mu \mathrm{m} ; \mathrm{C}, \mathrm{D}-20 \mu \mathrm{m} ; \mathrm{F}-\mathrm{H}-3 \mu \mathrm{m}$ 


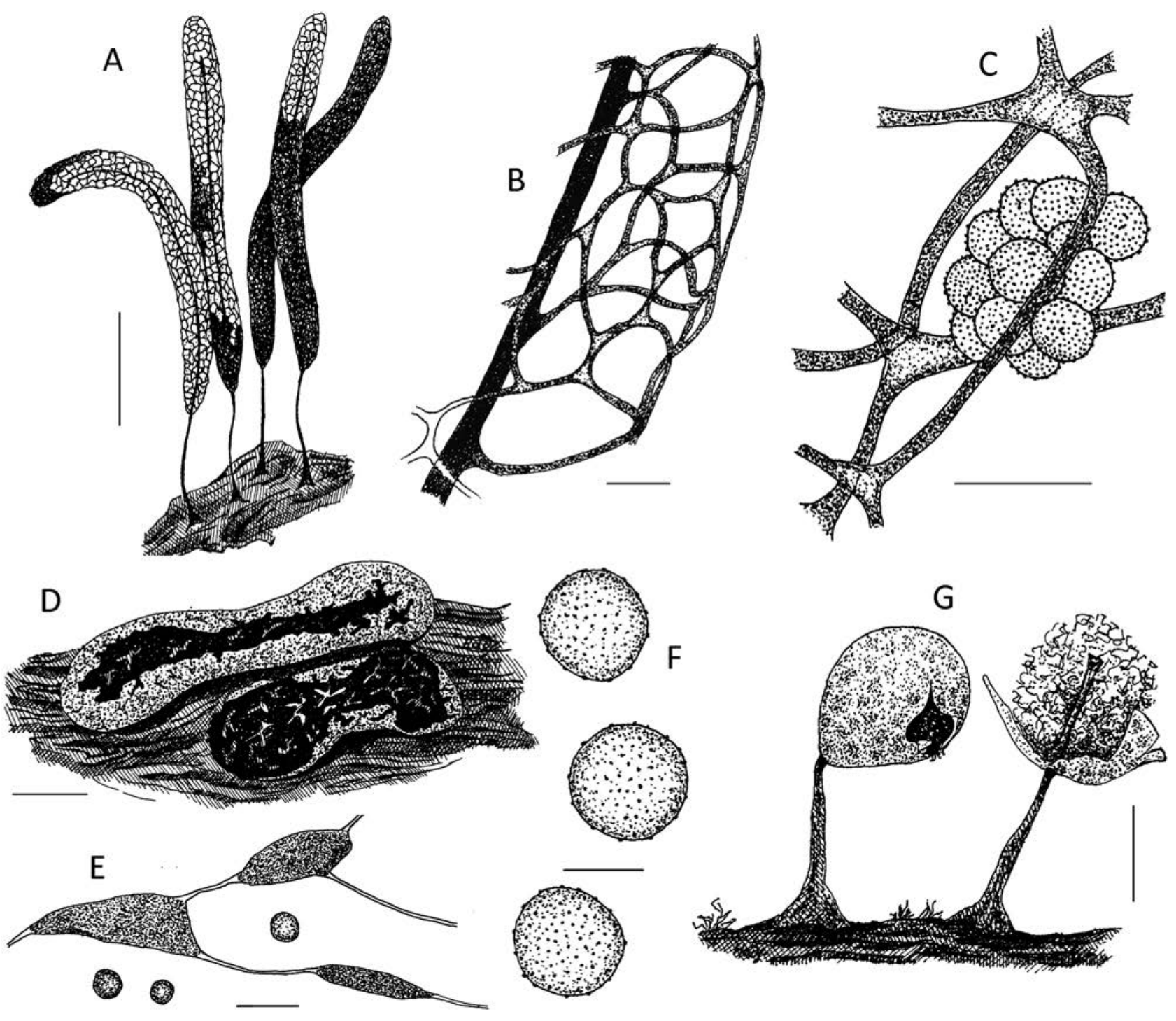

Figure 5 Drawings. A-C - Stemonitis uvifera T. Macbr. (462), A - sporophores, B - fragment of columella and capillitium, C - fragment of surface network and clustered spores; D-F - Physarum nitens (Lister) Ing (400), D - two plasmodiocarps, E - fragment of capillitium and spores, F - spores; G - Physarum penetrale Rex (22), two sporophores. Scale bars: A - 2 mm; B, E - $25 \mu \mathrm{m}$; C - $20 \mu \mathrm{m} ; \mathrm{D}-0.4 \mathrm{~mm}$; F - $5 \mu \mathrm{m}$; $\mathrm{G}-0.2 \mathrm{~mm}$

lime nodes, which holds its shape after spore dispersal. In Russia, it was found only in Krasnoyarsk Territory (Kosheleva 2007, Kosheleva et al. 2008), Vladimir Region (Mishulin 2018), and in the Far East (F.M. Bortnikov, Yu.K. Novozhilov, personal data). Physarum psittacinum Ditmar, in Sturm - 27 (356); 64f (1297); 78q (1187); 78r (1184); 78t (29); 78y (459); 110o (1472); 168d (81).

Physarum pusillum (Berk. et M.A. Curtis) G. Lister, in Lister $-29 \mathrm{~b}(232)$.

Physarum serpula Morgan - 63 (1251); 74 (1203).

Physarum spectabile Nann.-Bremek., Lado et G. Moreno 35d (95); 110e (1330); 110f (1315); 175b (894).

New for Moscow, Moscow and Tyumen Regions. This species is very similar to $P$. cinereum, but differs by dark-brown spores with light lines of dehiscence and well-developed warts. For the first time P. spectabile was recorded in the Central Forest Natural State Reserve (Nelidovo District, Tver Region) (Gmoshinskiy et al. 2017). This species is probably widely distributed in Russia.

Physarum vernum Sommerf., in Fries - 110e (1292).

Physarum virescens Ditmar, in Sturm - 78g (319); 78t (257); 78y (458).
Physarum viride (Bull.) Pers. - 29b (233, 236); 69a (1565); 78g (280-2); 89h (917); 136a (439); 159a (559); 160 (575); 166b (678); 174a (945-2, 947-2, 980).

Physarum viride var. aurantium (Bull.) Lister - 78g (309); 110k (1426); 174a (947-1).

Reticularia intermedia Nann.-Bremek. - 16a (824); 110g (912); 110m (1586-1); 124 (338).

New for Irkutsk and Murmansk Region.

Reticularia lycoperdon Bull. - 8 (610); 11 (608, 613); 15 (1594); 31 (761); 56 (204); 64c (860); 73b (876); 78w (269); 110e (1150); 110p (1618); 118 (5); 122 (487); 125 (476); 172 (171). New for Irkutsk Region.

Reticularia splendens Morgan - 20 (1583); 110f (1133); 132 (812); 142b (728).

New for Khabarovsk Territory and Republic of Karelia.

Stemonaria irregularis (Rex) Nann.-Bremek., R. Sharma et Y. Yamam., in Nannenga-Bremekamp, Yamamoto et Sharma (Fig. 4F) - 16b (1383); 29e (1612); 94d (111); 136b (1033); 168m (636).

New for Irkutsk and Tver Regions. 
Stemonitis axifera (Bull.) T. Macbr. - 7 (490, 493); 16d (932); 20 (1584); 23 (764); 26 (358); 29b (215, 219); 53b (163); 78f (321); 78g (313, 317); 78j (514, 524, 529); 78o (713); 78t (248, 259); 79 (423); 85d (886); 88 (1224, 1250, 1577); 89h (880, 923, 1276, 1591); 89j (1254-1); 100a (184); 100d (591); 109 (425); 110e (1334); 110f (1332); 110g (904, 908); 110h (830); 110j (1018-1); 110k (1425); 1101 (1414); 110m (1546); 110o $(1455,1458,1473,1477,1480) ; 110 \mathrm{p}$ (1624); 111 (1505-1); 113b (361); 116 (397); 117 (1416, 1528, 1529); 125 (472, 484); 131 (245); 135 (392); 142a (178); 142b (721, 722); 150 (546); 151 (497); 153 (544); 160 (541, 547); 161 (542); 166b (679); 168i (39)); 168j (586); 168k (536); 1681 (684); 168m (632, 6331); 168n (780, 783); 168p (1439, 1448, 1449); 168q (61); 168s (443); 169b (1355); 169c (1055, 1059, 1060, 1068, 1074, 1076 1083); 170 (1172, 1174); 174a (962, 977); 174b (1043, 1228, 1249); 175b (900); 176b (670); 179 (1285).

New for Khabarovsk Territory and Irkutsk Region.

Stemonitis axifera var. smithii (T. Macbr.) Hagelst. - 109 (381); 158 (539); 168m (637); 169c (1077, 1148); 174a (975).

Stemonitis flavogenita E. Jahn - 151 (540, 545); 155 (543); 168k (471).

New for Ryazan Region.

Stemonitis fusca Roth - 53b (162); 78g (279); 78y (410); 85g (770); 89j (1254-2); 94g (429); 109 (402); 110j (1018-2); 121 (820); 125 (473); 135 (418); 137b (1003); 168k (470); 168m (633-2); 168p (1452); 169b (1338); 170 (1321); 174a (976).

New for Tyumen Region.

Stemonitis fusca var. nigrescens (Rex) Torrend - 88 (1206-1). Stemonitis lignicola Nann.-Bremek. - 29b (239); 110e (1339); 121 (821); 134 (447); 142b (729); 145 (538); 166b (682); 168d (59, 60); 168f (143); 168r (58); 168s (440); 169c (1073, 1075, 1080).

Stemonitis pallida Wingate, in Macbride - 168n (781).

Stemonitis splendens Rostaf. - 3b (1578); 134 (398); 136a (391, 428); $137 \mathbf{b}$ (1011); 138 (901); 174b (1214).

New for Tyumen Region.

Stemonitis uvifera T. Macbr. (Fig. 4G-H, 5A-C) - 136c (1016). New for Russia (Primorye Territory).

Description. Sporophores stalked sporangia, clustered in large groups, long, cylindrical, erect or slightly dropping (Fig. 5A), rich rusty-brown; become dark-brown or black, semi-transparent after spore dispersal, 7-9 mm high; often join and form a structure resembling a pseudoaethalium. Peridium evanescent. Hypothallus well developed, continuous under a colony membranous shining, violet-brown. Stalk black, shining, from $1 / 4$ to $1 / 2$ of the total high, tapering upward, expanded below. Columella black, long, almost reaches the top of sporangium, in upper part curved and gradually transferred into a capillitium network. Capillitium forms a dark-brown to almost black net, with copper reflection. Inner network is very lax, arising from the columella by relatively few branches, often flattened, with membranous expansions in the axils. Surface net large-meshed (the mesh diameter is about 8-10 times the spore diameter), irregular, with many free ends (Fig. 5B-C). Spores dark-brown in mass; smoky-brown in transmitted light; united in clusters of 4-12 or more (Fig. 4G-H, 5C), often angular from pressure, occasionally free and then globose; uniformly warted on external surface of a group and almost smooth inside a cluster (Fig. 4H), 7-8 um in diam. Plasmodium white before fruiting (Martin \& Alexopoulos 1969).

Distribution. This is a very rare species. It is reported from USA, Canada, Germany (GBIF Secretariat 2019), India, Japan, Africa (Poulain et al. 2011a), and Spain (Lado 1994).

Notes. This is the only species from the genus Stemonitis with spores adhered in clusters. $S$, uvifera is closely related to species from the genus Symphytocarpus because of sporophores adhered in dense groups and spores joined into clusters (S. fusciformis Nann.-Bremek. et Härk. and S. syncarpus (Yamash.) Y. Yamam). All the latter species, however, differ by more heaped sporophores taking the almost aethaloid form. Moreover, S. fusciformis also differs with its black, expanded ends of capillitium, resembling Paradiacheopsis fimbriata (G. Lister et Cran) Hertel ex Nann.-Bremek. (Nannenga-Bremekamp \& Härkönen 1979). S. syncarpus differs by rounded fragments of peridium attached to the ends of capillitium, larger spores (12-13 $\mu \mathrm{m}$ vs. $7-8 \mu \mathrm{m}$ in S. wifera) and short stipes of sporangium (about $1 / 8$ of the total height) (Poulain et al. 2011a) Another species of Stemonitidales with clustered spores is Stemonaria rufipes Nann.-Bremek. et Y. Yamam. (NannengaBremekamp et al. 1984), characterised by short sporophores no more than $2.5 \mathrm{~mm}$ high (vs. $7-9 \mathrm{~mm}$ in $S$. wifera) and its oblong-oval shape (Lado et al. 2018).

Stemonitopsis hyperopta (Meyl.) Nann.-Bremek. - 40 (339); 78q (1181); 88 (1206-2); 1101 (1409); 110o (1474, 1483); 132 (803); 142b (730); 160 (549); 1681 (691); 168m (634); 168n (782, 784); 168p (1443, 1444); 171 (789); 174a (972); 174b (1036, 1048).

New for Ryazan and Tyumen Regions.

Stemonitopsis reticulata (H.C. Gilbert) Nann.-Bremek. et Y. Yamam., in Yamamoto et Nannenga-Bremekamp - 89d (746, 749, 750)

Stemonitopsis typhina (F.H. Wigg.) Nann.-Bremek. - 29b (210); 39 (347); 50 (1517); 52 (1190); 71 (878); $78 f$ (312); 78g (285, 326); 78o (712); 78q (1185); 78y (454); 88 (1223); 89d (748); 107a (154); 109 (411); 110e (1349); 110j (1019); 110o (1459, 1476, 1481); 110s (1116); 117 (1527-2, 1530); 125 (483); 136a (379, 438); 139 (1599); 1681 (688); 169c (1050, 1151); 174a (950, 967); 175b (899).

Stemonitopsis typhina var. similis (G. Lister) Nann.-Bremek. et Y. Yamam. - 78e (298).

Symphytocarpus amaurochaetoides Nann.-Bremek., in Ing et Nannenga-Bremekamp - 78m (511); 92 (1510).

Symphytocarpus flaccidus (Lister) Ing et Nann.-Bremek. 132 (808); 142b (719, 723, 727); 168g (200); 168j (587).

New for Murmansk Region.

Symphytocarpus impexus Ing et Nann.-Bremek. - 41 (345); 42 (446); 44 (365); 1681 (687).

New for Moscow and Moscow Region.

Trichia botrytis (J.F. Gmel.) Pers. - 1 (1281); 18 (499); 46-b (123); 53-b (161, 165); 89-h (914, 1259); 99 (54-1); 110-e (1346); 110-1 (1411); 110-p (1623); 132 (815).

New for Kaluga Region.

Trichia contorta (Ditmar) Rostaf. - 10 (585); 96b (581-1); 100d (594); 110e (1347); 1101 (1405); 110m (1549); 110p (1636). Trichia decipiens (Pers.) T. Macbr. (Fig. 1) - 16a (826); 16b $(1385,1397) ; 16$ c (1105); 17 (1595-1); 29e (1605); 33 (437); 34 (73-1); 35d (90, 102); 40 (344); 46b (125); 53b (169); 67c (135); 77a (51); 78b (291); 78d (152); 78e (297); 78i (407); 78q (1168); 85a (115); 85e (1025, 1358); 88 (1191); 89d (756); 89e (1268, 1278); 89h (919, 1265, 1273); 91 (1238); 94b (56); 94d (50, 53 , 113); 98 (55); 99 (54-2); 100c (654); 100d (592); 102 (71); 107a $(75,85,86) ; 107$ c (372); 110 c $(615,645) ; 110$ e $(1120,1134,1138,1141,1143-2,1306,1316) ; 110 f(1303-1$, 1313, 1325); 110h $(835,1366,1371,1400) ; 110 j(1021,1022) ;$ 110k (1420); 1101 (1413); 110m (1550); 110o (1485); 110p (1620, 1628); 110r (1112); 111 (1488, 1504, 1505-2, 1592-1); 117 (1525); 167 (1639); 168e (52); 168m (699); 169c (1057, 1071, 1147); 174a (944, 965).

New for Irkutsk Region.

Trichia favoginea (Batsch) Pers. - 5 (1569); 28 (626); 29e (1613, 1614); 34 (73-2); 35b (47); 78g (281); 78u (262); 79 (396); 85e (1024); 89d (752-1); 89f (887); 110a (43, 49); 110c (650); 110d (651); 110e (1142, 1323); 110g (905); 110h (836, 1378); 110k (1429); 110p (1631); 110s (1123); 111 (1490, 14952, 1499-2, 1502, 1503); 115 (146, 183); 117 (1526); 161 (569); 166b (683); 168m (622-2, 639-2); 168q (46); 174a (942); 175b (890); 176a (769); 176b (672).

Trichia lutescens (Lister) Lister - 24 (510); 78k (995).

New for Krasnodar Territory.

Trichia persimilis P. Karst. - 16b (1386); 16c (1089-2, 1099, 1102); 18 (498); 30 (732); 78c (137); 78d (147); 78i (377); 89d (752-2); 94d (13-2, 45); 107a (88); 110a (42); 110e (1327); 110f (1348); 112a (363); 137b (1001); 167 (1431); 169c (1049, 1052).

New for Irkutsk and Kaluga Regions.

Trichia scabra Rostaf. - 12 (607, 612, 665); 13 (772); 16c (1098, 1103); 16d (930); 35d (97); 46b (124); 52 (1207); 78g (325); 78j (532); 78t (256); 88 (1216); 89h (1294); 96a (448); 102 (72); 110e (1129, 1333); 110m (1538, 1540, 1586-2); 110p (1617); 110s (1153); 111 (1506-2, 1592-2, 1593-2); 117 (1523); 166b (677); 168p (1447); 168q (48).

New for Irkutsk Region.

Trichia varia (Pers. ex J.F. Gmel.) Pers. - 12 (609, 666); 13 (771); 16a (825); 16c (1104); 17 (1595-2); 20 (1390, 1392); 22 
(763); 29c (192); 29d (371); 29e (1606); 30 (733); 35d (103); 36 (199); 39 (351); 46b (126); 53b (177); 57 (1188); 64a (861-2, 862-2); 76 (993); 77d (130); 78d (160); 78h (453); 78i (387); 78k $(520,527,697) ; 78 w(271) ; 83 \mathrm{a}(759) ; 85 \mathrm{a}(116) ; 85 \mathrm{~b}(872) ; 85 \mathrm{e}$ $(1357,1362) ; 89 d$ (752-3, 757); 89h (1269); 94d (1580); 94e (141, 149, 155); 94i (852); 94j (1581); 96b (581-2); 110c (618); 110e $(1128,1131,1136,1139,1295,1305,1326,1341-2) ; 110 f$ (1303-2, 1304, 1343, 1351-2); 110g (926); 110h (1367, 1403-2); 1101 (1408); 110o (1453); 110p (1621, 1635); 110s (1117, 1144); 111 (1497); 125 (482); 169c (1058); 175b (889).

New for Irkutsk Region.

Tubifera applanata Leontyev et Fefelov - 29-b (211); 29-e (1604); 29-f (212); 78-g (287); 89-d (735); 142-a (175); 142-b (718).

New for Moscow, Moscow Region and Republic of Karelia.

Tubifera ferruginosa (Batsch) J.F. Gmel. - 37 (341); 45a (2); 46b (336); 65a (630); 77a (3); 781 (513); 78n (869); 78t (258); 78y (434); 89f (695); 89h (918); 96a (436); 107a (91); 107d (419); 109 (380, 390); 110c (621); 110e (1553); 110h (841, 1375); 110o (1470); 117 (1531); 118 (4); 132 (806); 166b (681); 168g (203); 168k (534); 168m (625); 168n (776); 168p (1442).

List of undetermined specimens

To the genus level: Arcyria sp. - 53b (164); 110e (1287-1); 138 (902). Diderma sp. - 77b (74); 110c (643). Didymium sp. - 88 (1225). Licea sp. - 110h (847); 132 (814). Reticularia sp. - 78k (465). Stemonaria sp. - 12 (657, 659); 78u (264); 176b (668). Stemonitis sp. - 16c (1101); 78t (250); 78u (261); 110h (827).

To the order level: Stemonitidales - 45f (57); 78g (180); 84 (394); 110h (1363, 1365). Physarales - 170 (1169).

Not identified to the order level: $12(611,658) ; 16 c$ (1155); 64e (1231); 78o (714); 78q (1167, 1178); 85a (132); 89d (747); 110e (1337); 110h (845, 1404); 123 (408); 125 (474); 128 (488); 136c (999); 137b (990, 992, 1006, 1010); 148b (598); 148c (597); 153 (467); 166b (680); 168d (62); 174a (952, 954).

\section{Current state of the Myxomycetes collection}

The Myxomycetes collection of the CYN includes 1715 specimens of sporophores belonging to 142 species of myxomycetes from 35 genera, 11 families, and 6 orders. Two species, Stemonitis wifera and Fuligo intermedia, were recorded for the first time in Russia. The majority of the specimens in collection belongs to the Trichiales (654 specimens / 38.7\% of total), Physarales (431 / $25.5 \%$ ), and Stemonitidales (331 / $25.5 \%)$. There are fewer specimens from the Cribrariales (238 / $14.1 \%$ ), Ceratiomyxales (31 / $1.8 \%$ ), and Echinosteliales (3 specimens / $0.2 \%$ ). Moreover, 27 specimens were not identified (see the list of undetermined specimens). Taxonomic structure of the Myxomycetes collection of the CYN is presented in Table 2.

The greatest contribution to the collection establishment was made by O.M. Germant (614 deposited specimens), E.A. Dunayev (267), N.N. Kotelenets (248), T.N. Barsukova (117), V.I. Gmoshinskiy (64), A.N. Bragin (63). During the whole period of the collection existence, only O.M. Germant and E.A. Dunayev were regularly depositing new material into it, while the contribution from other collectors was irregular.

Almost all specimens in the Myxomycetes collection of the CYN were collected in Russia. Many findings presented in the collection are new for the regions (Table 3).

Despite the fact that the specimens in the collection were not fixed on paper tray in matchboxes before the revision and that the age of some of them exceeded 20 years, most of the material (1331 specimens) was kept in satisfactory, good,
Table 2. Taxonomic structure of Myxomycetes collection of the CYN. Numbers in parentheses show number of species in taxon

\begin{tabular}{|c|}
\hline $\begin{array}{l}\text { Order Family } \\
\quad \text { Genus }\end{array}$ \\
\hline $\begin{array}{l}\text { Ceratiomyxales (1) } \\
\text { Ceratiomyxaceae (1) } \\
\text { Ceratiomyxa (1) }\end{array}$ \\
\hline $\begin{array}{l}\text { Echinosteliales (1) } \\
\text { Clastodermataceae (1) } \\
\text { Clastoderma (1) }\end{array}$ \\
\hline $\begin{array}{c}\text { Cribrariales (25) } \\
\text { Cribrariaceae (15) } \\
\text { Cribraria (14) } \\
\text { Lindbladia (1) } \\
\text { Dictydiaethaliaceae (1) } \\
\text { Dictydiaethalium (1) } \\
\text { Reticulariaceae (7) } \\
\text { Lycogala (2) } \\
\text { Reticularia (3) } \\
\text { Tubifera (2) } \\
\text { Liceaceae (2) } \\
\text { Licea (2) }\end{array}$ \\
\hline $\begin{array}{c}\text { Physarales (59) } \\
\text { Physaraceae (41) } \\
\text { Badhamia (6) } \\
\text { Craterium (4) } \\
\text { Fuligo (5) } \\
\text { Leocarpus (1) } \\
\text { Physarum (25) } \\
\text { Didymiaceae (18) } \\
\text { Diachea (1) } \\
\text { Diderma (4) } \\
\text { Didymium (11) } \\
\text { Lepidoderma (1) } \\
\text { Mucilago (1) }\end{array}$ \\
\hline $\begin{array}{l}\text { Stemonitidales (26) } \\
\text { Stemonitidaceae (26) } \\
\text { Amaurochaete (1) } \\
\text { Collaria (1) } \\
\text { Comatricha (1) } \\
\text { Enerthenema (1) } \\
\text { Lamproderma (3) } \\
\text { Macbrideola (1) } \\
\text { Stemonaria (1) } \\
\text { Stemonitis (7) } \\
\text { Stemonitopsis (3) } \\
\text { Symphytocarpus (3) }\end{array}$ \\
\hline $\begin{array}{c}\text { Trichiales (30) } \\
\text { Arcyriaceae (16) } \\
\text { Arcyodes (1) } \\
\text { Arcyria (12) } \\
\text { Perichaena (3) } \\
\text { Trichiaceae (14) } \\
\text { Hemitrichia (4) } \\
\text { Metatrichia (2) } \\
\text { Trichia (8) }\end{array}$ \\
\hline
\end{tabular}

or excellent condition (levels III to V according to Table 1). Thus, a fact of these species record could be confirmed by morphological features. Only 384 specimens were poorly preserved or were completely without sporophores (levels I and II), which accounted for $22.4 \%$ of the whole collection. It is interesting to note that the level of preservation of the material did not depend on the storage time in the herbarium (Fig. 6). On the contrary, the largest number of specimens in perfect condition were obtained in the early period of collection. Hence, it can be assumed that during storage the 
morphological features of sporophores were slightly changed, if changed at all. Moreover, we do not have information about the condition in which specimens were initially obtained. A significant part of them was most likely far from being perfectly preserved at the time of gathering in the field. It should be noted that despite the lack of the collection processing by any special chemicals (such as mercuric chloride) or prefreezing, only a few specimens had traces of insect damage. Representatives of the genera Badhamia and Reticularia were affected by insects the most.

The specimen preservation in different genera was also similar. The largest proportion of heavily damaged sporophores was related to the Physarales. Representatives of the genera Badhamia and Physarum, which have relatively small and very fragile sporophores, suffered the most from mechanical damage (Fig. 7). Also the specimens belonging to the genus Comatricha were hardly-damaged due to non-fixed storage of such fragile sporophores. Specimens from the genus Tubifera were also damaged, including the sporophore tops, which are very important for identification. A slightly smaller part of heavily damaged sporangia was noted in the Cribrariales, Stemonitidales, and Ceratiomyxales. Specimens from the Trichiales (Trichia, Hemitrichia, Arcyria) were preserved the best due to the fact that the majority of their representatives have a sufficiently elastic peridium, which damages less by mechanical impact. Colonies of the genus Didymium, as a rule, are light color immediately after maturation because of lime on the sporophore surface, which makes them clearly visible on leaf litter. However, under the influence of precipitation, lime might wash off, so sporangia turn black and almost invisible on the substrate. As a result, they are often skipped during field collection. At the same time, sporophores are initially strong in dry condition and relatively serious impact is required to damage them. Thus, when species of this genus are collected in good condition, they are almost not damaged during storage, and it can explain a high proportion of well preserved specimens of Didymium species.

We can conclude that critical revisions of old collections can provide valuable information on the myxomycete distribution.

\section{ACKNOWLEDGEMENTS}

We are grateful to members of the Interdepartmental Electron Microscopy Laboratory (Faculty of Biology, Lomonosov MSU) for their technical support and N.I. Kireeva for drawings of specimens. Field work was supported by Russian Foundation for Basic Research (project 18-04-01232 A). Identification of the material was performed within the framework of the State task of MSU, part 2 (topic number AAAA-A16-116021660084-1). Work of the first author was supported by Moscow State University Grant for Leading Scientific Schools "Depository of the Living Systems" within frame of the MSU Development Program.
Table 3. Distribution of species and specimens by regions in the Myxomycetes collection of CYN

\begin{tabular}{lccc}
\hline Region & $\begin{array}{c}\text { Number of } \\
\text { specimens }\end{array}$ & $\begin{array}{c}\text { Number of } \\
\text { deposited } \\
\text { species }\end{array}$ & $\begin{array}{c}\text { Number of } \\
\text { new species } \\
\text { for the region }\end{array}$ \\
\hline Moscow and Moscow Region & 1112 & 122 & 11 \\
Tver Region & 191 & 55 & 3 \\
Tyumen Region & 112 & 49 & 27 \\
Ryazan Region & 56 & 30 & 12 \\
Irkutsk Region & 50 & 25 & 24 \\
Murmansk Region & 50 & 29 & 7 \\
Primorye Territory & 50 & 27 & 1 \\
Crimean Peninsula & 34 & 14 & 1 \\
Republic of Karelia & 18 & 11 & 3 \\
Khabarovsk Territory & 17 & 13 & 6 \\
Krasnodar Territory & 8 & 7 & 1 \\
Pskov Region & 4 & 3 & 3 \\
Kaluga Region & 3 & 3 & - \\
Altai Territory & 2 & 1 & 1 \\
Astrakhan Region & 2 & 2 & - \\
Yaroslavl Region & 2 & 2 & - \\
Altai Republic & 1 & 1 & - \\
Vladimir Region & 1 & 1 & - \\
Kingdom of Cambodia & 1 & 1 & \\
No georeference & 1 & 1 & \\
\hline
\end{tabular}

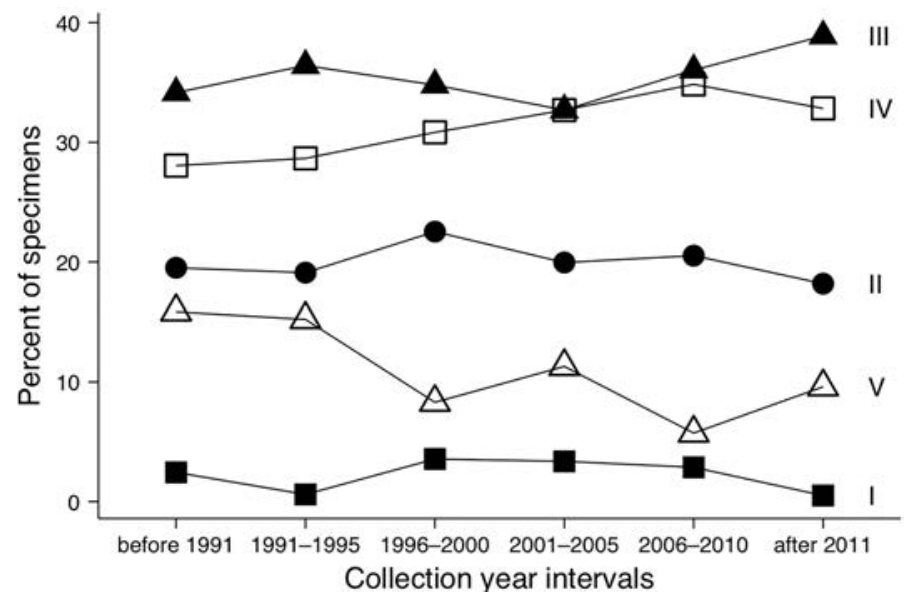

Figure 6 Preservation level of specimens collected in different years and deposited in the CYN collection. Description of preservation levels see in Table 1

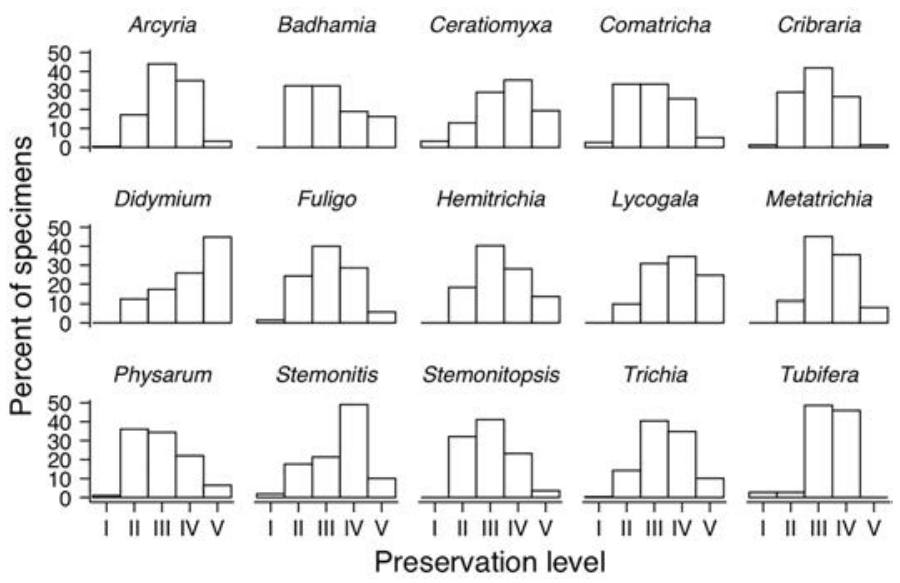

Figure 7 Preservation level of most abundant genera (with number of specimens more than 30) on a five-point scale (see Table 1) in the Myxomycetes collection of CYN 


\section{LITERATURE CITED}

Barsukova, T.N. \& E.A. Dunaev 1997. An annotated list of slime moulds (Myxomycota) from Moscow Region. Mikologiya i fitopatologiya 31(2):1-8 (in Russian with English summary). [Барсукова Т.Н., Аунаев Е.А. 1997. Аннотированный список слизевиков (Myxomycota) Mocковской области // Микология и фитопатология. T. 31, вып. 2. С. 1-8].

dela Cruz T.E.E., R.V. Kuhn, A.O.M. Javier, C.M. Parra \& T.H. Quimio 2009. Status of the Myxomycete Collection at the UPLB-Museum of Natural History (UPLB-MNH) Mycological Herbarium. Philippine Journal of Systematic Biology 3(1):97-111.

Dunayev, E.A. 2017. Young Naturalists Club of Scientific Research Zoological Museum of Moscow State University. KMK, Moscow, 81 pp. (in Russian). [Аунаев E.A. 2017. Кружок юных натуралистов Научно-исследовательского Зоомогического музея МГУ (КЮН ЗМ МГУ). Москва: KMK. 81 c.].

Dunayev, E.A. \& T.N. Barsukova 2002. Collection of slime moulds (Myxomycota) of Young Naturalists Club of Zoological Museum MSU. Current mycology in Russia. Abstracts of First Congress of Russian Mycologists, p. 132, National Academy of Mycology, Moscow (in Russian). [Аунаев Е.А., Барсукова Т.Н. 2002. Комлекция слизевиков (Myxomycota) Кружка юных натуралистов Зоомузея МГУ // Современная микология в России. Первый съезА микологов в России. Тезисы докладов. М.: Национальная академия микологии. С. 132].

GBIF Secretariat 2019. GBIF Backbone Taxonomy. Available from: https://doi.org/10.15468/39omei. Last accessed 20.05.2020.

Gmoshinskiy, V.I., N.Yu. Buchtoyarova \& A.V. Matveev 2017. First record of Physarum spectabile (Myxomycetes) in Russia. Botanica Lithuanica 23(2):107-110.

Gmoshinskiy, V.I., A.V. Matveev \& E.A. Dunaev 2018. Collection of myxomycetes of Young Naturalists Circle of Zoological Museum of Moscow State University. Lomonosov Moscow State University. Occurrence dataset https://doi.org/10.15468/ zmmwmj (Last accessed 18.04.2020).

Kosheleva, A.P. 2007. Myxomycetes of "Stolby" Reserve (East Sayan): taxonomical composition and ecology ( $\mathrm{PhD}$ thesis). Saint-Petersburg, 160 pp. (in Russian). [Кошелева А.П. 2007. Миксомицеты заповедника «Столбы» (Восточный Саян): таксономический состав и экология (Аиссертация на соискание уч. ст. к.б.н.) Санкт-Петербург: БИН РАН. 160 с.].

Kosheleva, A.P., Yu.K. Novozhilov \& M. Schnittler 2008. Myxomycete diversity of the State Reserve "Stolby" (south-eastern Siberia, Russia). Fungal Diversity 31:45-62.

Lado, C. 1994. A checklist of Myxomycetes of the Mediterranean countries. Mycotaxon 52(1):117-185.

Lado, C. 2005-2020. An on line nomenclatural information system of Eumycetozoa. Real Jardín Botánico, CSIC. Madrid, Spain. http://www.nomen.eumycetozoa.com (Last accessed 23.01.2020).

Lado, C. \& U. Eliasson 2017. Taxonomy and systematics: current knowledge and approaches on the taxonomic treatment of Myxomycetes. In: Myxomycetes: biology, systematics, biogeography, and ecology (S.L. Stephenson, C. Rojas, eds), pp. 205-252, Academic Press, London.

Lado, C., A. Estrada-Torres \& C. Rojas 2018. New records of genera and species of Myxomycetes (Amoebozoa) from the Neotropics. Checklist 14(3):509-518.
Lavrov, N.N. 1929. Materials for the Siberian slime moulds flora: 2. Slime moulds of Northern and Central Altai. Izvestiya Tomskogo otdeleniya Russkogo botanicheskogo obshchestva 4-5:84-100 (in Russian). [Аавров Н.Н. 1929. Материамы Аля фморы слизевиков Сибири: 2. Слизевики Северного и Центрального А^тая // Известия Томского отАеления Русского ботанического общества. T. 4-5. C. 84-100].

Matveev, A.V., F.M. Bortnikov, V.I. Gmoshinsky \& Yu.K. Novozhilov 2016-2020: Myxomycetes of Russia. Web application. Available from: http://myxomycetes.org/russia. Last accessed 17.03.2020.

Martin, G.W. \& C.J. Alexopoulos 1969. The Myxomycetes. University Iowa Press, Iowa City, 561 pp.

Mishulin, A.A. Data on myxomycetes of Vladimir Region. In: Materialy mezhdunarodnogo molodezhnogo nauchnogo foruma "Lomonosov 2018". MSU, Moscow (in Russian). Available from: https://lomonosov-msu.ru/archive/Lomonosov_2018/data/12720/68511_uid243842_report.pdf. Last accessed: 10.02.2020 (in Russian). [Мишулин A.A. 2018. Аанные о биоте миксомицетов ВАадимирской области // Материалы межАународного молодежного научного форума « Аомоносов 2018». Москва: МГУ.

Nannenga-Bremekamp, N.E. \& M. Härkönen 1979. Symphytocarpus fusiformis (Myxomycetes) a new species from Finland. Proceedings van de Koninklijke Nederlandse Akademie van Wetenschappen Section C 82(3):371-374.

Nannenga-Bremekamp, N.E., Y. Yamamoto \& R. Sharma 1984. Stemonaria, a new genus in the Stemonitaceae and two new species of Stemonitis (Myxomycetes). Proceedings van de Koninklijke Nederlandse Akademie van Wetenschappen Section C 87:449-469.

Novozhilov, Yu.K. 1993. Flora of the fungi of Russia. Division Slime moulds. Issue 1. Class Myxomycetes. Nauka, Saint-Petersburg, 288 pp. (in Russian). [Новожилов Ю.К. 1993. Определитель грибов России. ОтАел Слизевики. Вып. 1. К^асс Миксомицеты.Санкт-Петербург: Наука. 288 с.].

Novozhilov, Yu.K., I.V. Zemlianskaia, M. Schnittler \& K.A. Fefelov 2003. An annotated checklist of the myxomycetes of the northwestern Caspian lowland. Mikologiya i fitopatologia 37(6):53-65.

Novozhilov, Yu.K. 2005. Myxomycetes of Russia: Taxonomic composition, ecology and geography (Doct. Diss.). Saint-Petersburg, 377 pp. (in Russian). [Новожилов Ю.К. 2005. Миксомицеты (класс Мухотусеtеs) России: Таксономический состав, экология и география (Аиссертация на соискание уч. ст. А.б.н.). Санкт-Петербург: БИН PAH, 377 c.].

Novozhilov, Yu. K., M. Schnittler \& S.L. Stephenson 2009. Biogeographical and ecological patterns of myxomycete assemblages in high-latitude and arid areas. In: Species and communities in extreme environments. Festschrift to the 75 th Birthday of Academican Yuri Ivanovich Chernov (O.L. Makarova, A.B. Babenko, S.I. Golovatch \& L.D. Penev, eds), pp. 191-207, Pensoft Publishers, Sofia, Moscow.

Novozhilov, Yu.K., M. Schnittler, A.V. Vlasenko \& K.A. Fefelov 2010. Myxomycete diversity of the Altay Mountains (southwestern Siberia, Russia). Mycotaxon 111:91-94.

Poulain, M., M. Meyer \& J. Bozonnet 2011a. Les Myxomycétes. Tome 1. Guide de détermination. Fédération mycologique et botanique Dauphiné-Savoie, Sévrier, France, 568 pp.

Poulain, M., M. Meyer \& J. Bozonnet 2011b. Les Myxomycétes. Tome 2. Planches. Fédération mycologique et botanique Dauphiné-Savoie, Sévrier, France, 544 pp. 
Schnittler, M., N.H.A. Dagamac \& Yu.K. Novozhilov 2017. Biogeographical patterns in Myxomycetes. In: Myxomycetes: biology, systematics, biogeography, and ecology (S.L. Stephenson \& C. Rojas, eds), pp. 299-331, Academic Press, London.

Stephenson, S.L. \& H. Stempen 2000. Myxomycetes: A Handbook of Slime Molds. Timber Press, Inc., Portland, 183 pp.

Sizova, T.P. \& Yu.A. Titova 1985. Slime fungi on the territory of Zvenigorodskaya Biological station (Biological department of the Moscow State University). Byulleten' Moskovskogo obshchestva ispytatelei prirody. Otdel biologicheskii 90(2):113-117 (in Russian with English abstract). [Сизова Т.П., Титова Ю.А. 1985. Миксомицеты Звенигородской биологической станции Московского университета / / Бюлметень Московского общества испытателей природы. ОтАел биологический. Т. 90, № 2. C. 113-117].
Wieczorek, J., D. Bloom, R. Guralnick, S. Blum, M. Döring, R. Giovanni, T. Robertson \& D. Vieglais 2012. Darwin Core: An Evolving Community-Developed Biodiversity Data Standard. PLOS ONE 7(1):e29715.

Zemlianskaia, I.V. 2003. Myxomycetes of steppes and deserts of lower Volga region (PhD thesis). Volgograd, Saint-Petersburg, 213 pp. (in Russian). [Землянская И.В. 2003. Миксомицеты степей и пустынь Нижнего Поволжья (Аиссертация на соискание уч. ст. к.б.н.). Волгоград; Санкт-Петербург: ВолгограАский государственный медицинский университет. 213 с.]. 